\title{
Patienter sur les places et foncer dans les rues. Quand les coursiers occupent l'espace public
}

Waiting in the squares and rushing through the streets. When couriers occupy the public space

\section{Émilie Aunis et Hélène Stevens}

\section{OpenEdition}

Journals

Édition électronique

URL : https://journals.openedition.org/itti/1839

DOI : $10.4000 /$ itti. 1839

Éditeur

Université de Poitiers

Référence électronique

Émilie Aunis et Hélène Stevens, «Patienter sur les places et foncer dans les rues. Quand les coursiers occupent l'espace public », Images du travail, travail des images [En ligne], 11 | 2021, mis en ligne le 01 septembre 2021, consulté le 18 février 2022. URL : http://journals.openedition.org/itti/1839 ; DOI https://doi.org/10.4000/itti.1839

Ce document a été généré automatiquement le 18 février 2022.

Images du travail, travail des images 


\section{Patienter sur les places et foncer dans les rues. Quand les coursiers occupent l'espace public}

Waiting in the squares and rushing through the streets. When couriers occupy the public space

\section{Émilie Aunis et Hélène Stevens}

Parmi les travailleurs des plateformes numériques, ils sont assurément les plus visibles. Contrairement aux «travailleurs du clic» invisibilisés derrière leur ordinateur depuis leur domicile (Casilli, 2019), les coursiers travaillent dans l'espace public. On peut ainsi les apercevoir dans les grandes villes - facilement repérables par leur équipement aux couleurs et logo de la plateforme avec laquelle ils travaillent - attendre sur des places, s'élancer à toute vitesse dans les rues, récupérer des commandes chez les restaurateurs, sonner au bas des immeubles des clients.

«La rue est [leur] usine» comme l'affirme à juste titre le slogan de livreurs mobilisés pour défendre leurs droits et revendiquer de meilleures conditions de travail et de rémunération (photo 1). 
Photographie 1. " La rue est notre usine ». Manifestation des coursiers parisiens

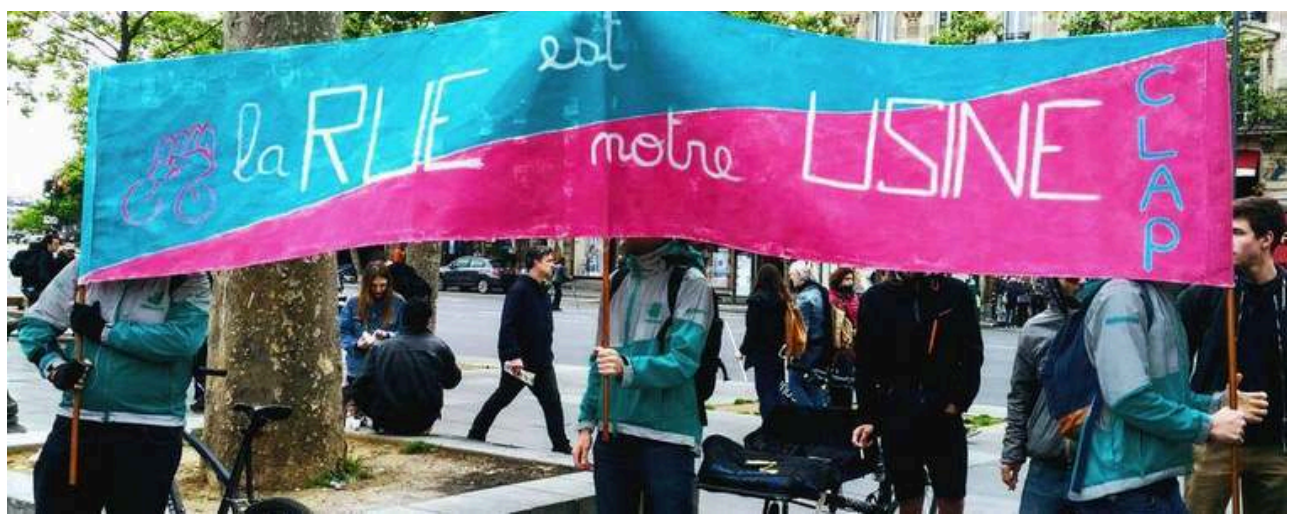

Source. Page Facebook du CLAP, Collectif des Livreurs Autonomes de Paris, Paris, mai 2017.

2 Pour autant, il ne suffit pas de travailler dans la rue pour être vu et de fait, certaines « caractéristiques [du travail] restent "invisibles" ( (Soares, 2011, 233). De même que pour d'autres travailleurs dans la rue, leur visibilité ne révèle pas mécaniquement au témoin la pénibilité des tâches réalisées, les compétences mises en œuvre, les formes de sociabilités ou encore la complexité des rapports sociaux qui se nouent.

Alors que les coursiers occupent l'espace public, sont apercevables par tous, que voit-on finalement d'eux ? Et qu'est-ce qui reste inaperçu malgré leur visibilité ? À partir d'une enquête de terrain mêlant observations, entretiens et analyse de données webographiques (cf. encadré 1), nous chercherons à saisir ce qui se joue dans les deux temps constitutifs de l'activité de coursier : celui de l'attente et celui de la course. Après avoir montré qu'un ensemble de stratégies, compétences et formes de sociabilités échappent à l'œil nu du passant, nous analyserons le double mouvement qui conduit les coursiers à se rendre discrets dans l'activité ou visibles par la mobilisation.

\section{Encadré 1. Des coursiers visibles mais parfois insaisissables pour l'enquêtrice}

L'analyse développée repose sur des séquences d'observation, 24 entretiens individuels avec des coursiers et des entretiens collectifs avec sept travailleurs sans-papiers ${ }^{1}$. La population des coursiers est hétérogène, et si l'accès aux étudiants pratiquant l'activité en parallèle de leurs études a été plutôt aisée, des difficultés ont été rencontrées pour accéder aux coursiers étrangers, dont une partie, « sans-papiers », est prise dans des pratiques illégales mais répandues de « sous-location » de comptes. Des démarches ont d'abord été entreprises en abordant directement ces travailleurs aux abords des restaurants devant lesquels ils attendent des courses. Mais ces approches directes se sont avérées infructueuses en raison parfois des difficultés langagières et plus largement de la distance sociale et genrée entre l'enquêtrice et les enquêtés, ainsi que de la défiance à l'égard de l'enquête sociologique, susceptible d'être apparentée à l'enquête policière. Afin de contourner ces obstacles, une première prise de contact avec une association locale d'aide aux migrants n'a pas permis d'ouvrir le terrain. D'autres associations ont ensuite été sollicitées et ont facilité la rencontre de trois travailleurs sans-papiers. Enfin, nous avons pu nous entretenir, par l'intermédiaire d'organisations syndicales, avec quatre coursiers sans-papiers, dans le cadre de leur mobilisation durant l'été 2020 contre la plateforme Frichti 
avec laquelle ils travaillaient et dont ils avaient été exclus.

Les photographies mobilisées dans l'article témoignent de ces difficultés : celles réalisées par les chercheurs ${ }^{2}$ l'ont souvent été "à la dérobée », de loin avec un téléobjectif ou lorsque les coursiers étaient de dos (permettant aussi de respecter leur anonymat). Par ailleurs, elles ont essentiellement pu être prises lors des temps d'attente de commandes, lorsque les travailleurs sont à l'arrêt, et très peu durant les temps de livraison durant lesquels ils sont en mouvement, ce qui réclame de la part du photographe de trouver un poste d'observation stratégique pour saisir leurs passages extrêmement furtifs (par exemple sur des axes régulièrement empruntés par les coursiers et permettant de « voir sans être vu »position d'enquête qui rappelle non sans ironie la thématique du numéro) et de maîtriser des compétences techniques pour capter la vitesse. Ces deux conditions n'ayant pu être satisfaites, le corpus de photos est réduit et limité.

Enfin, il faut souligner que ces photos de chercheurs contrastent avec celles prises par les coursiers eux-mêmes dans le cadre de leur mobilisation (cf. photos 1 et 6 à 9). Celles-ci, disponibles sur les sites des collectifs ou organisations syndicales de défense des droits des coursiers à vélo, sont l'objet d'une mise en scène : les coursiers posent de face, parfois le poing levé ou avec une banderole, et représentent ainsi la force de leur collectif.

\section{Aperçus sur les places : attendre et déjouer l'incertitude, seul ou en groupe}

\subsection{Tuer l'attente}

4 Le travail des coursiers à vélo se divise en différentes séquences que l'on peut schématiquement rassembler en deux principales activités : attendre une commande de repas ; livrer cette commande. La première consiste ainsi à patienter jusqu'à ce qu'une course leur soit attribuée sur leur téléphone via l'application de la plateforme numérique ou que le restaurateur ait fini de préparer la commande. Pour ces travailleurs indépendants payés à la course (cf. encadré 2 ), ce temps - variable de quelques minutes à quelques heures selon le jour, l'horaire mais aussi le nombre de coursiers connectés - ne fait l'objet d'aucune rémunération, cette dernière débutant au moment de l'acceptation de la commande pour se terminer dès la livraison effectuée. Il est ainsi largement subi par les livreurs. Loin d'être envisagé comme un temps de pause, de «reprise de soi » dans le cadre d'une activité intense (Hatzfeld, 2002), il est pensé comme du temps perdu, durant lequel on ne gagne rien alors qu'on reste mobilisé pour le travail, le corps en tension (Lemozy, 2019, 3-5), préoccupé de se voir attribuer rapidement une nouvelle commande :

"Entre deux courses des fois ça m'arrive de pas avoir de courses, du coup je suis obligé

d'attendre et... ouais c'est... tu perds des sous!»

$\mathrm{Abdou}^{3}, 20$ ans, sans diplôme, Sénégalais, Uber Eats et Deliveroo, $\sim 40 \mathrm{~h} /$ semaine

Entretien réalisé à Poitiers, le 29/05/2019.

«Hier je me suis connecté 4 heures il me semble... [il regarde sur son téléphone], alors

hier je me suis connecté 4 heures, j'ai fait 2 heures 30 de vélo.

- Parce qu'il y a beaucoup de temps d'attente tu veux dire?

- Parce qu'il y a du temps d'attente, parce que tu attends au restaurant et que même si tu attends 5 minutes, 5 minutes fois 10, ça fait tout de suite... une heure de perdue donc le 
temps de connexion... ok bon si c'est le temps où je me rends disponible pour Deliveroo, ça je peux pas le nier, c'est sûr que maintenant que je suis à 11 heures, je suis super assidu, j'ai envie de faire de l'argent donc oui c'est l'heure où vraiment je suis disponible pour Deliveroo, ça c'est sûr...»

Aloïs, 22 ans, bac éco ES, trois mois de fac éco, Deliveroo et Uber Eats, $~ 48$ h/sem.

Entretien réalisé à Poitiers, le 06/05/2019.

Encadré 2 : Conditions d'accès à l'activité et statut d'emploi des coursiers

Sur le plan juridique, les coursiers à vélo exercent l'activité sous le statut de microentrepreneurs, en tant que travailleurs indépendants via des contrats de prestations avec les plateformes. Cinq conditions principales sont exigées de la part des principales plateformes pour devenir coursier : être âgé d'au moins 18 ans, posséder une pièce d'identité valable dans l'Union européenne, avoir son entreprise ou microentreprise, disposer d'un vélo ou d'un mode de transport particulier et enfin posséder un smartphone récent et un forfait mobile. Aucune qualification ou expérience professionnelle n'est exigée et les modalités de sélection sont réduites à une inscription rapide sur internet et parfois quelques réponses à des questions standardisées lors d'un appel téléphonique. Cette activité est donc caractérisée par des conditions d'accès peu restrictives qui ont amené des personnes rencontrant des difficultés d'accès au marché du travail classique, du fait de manque de qualifications ou de discriminations, à l'exercer. Plus encore, parce que ces conditions d'accès sont peu contrôlées, elles ont constitué une occasion de ressources pour des travailleurs sans-papiers. Si ces derniers ne peuvent légalement pas avoir le statut d'autoentrepreneur, ils se saisissent néanmoins de cette activité économique pour gagner un revenu : ils négocient avec un travailleur en situation régulière, de nationalité française ou non, pour sous-louer son compte ${ }^{4}$. En contrepartie, ce dernier leur prélève un pourcentage de leur chiffre d'affaires variable selon les cas de figure, oscillant selon les sources entre 30 et $50 \%$.

En dépit de leur statut de travailleurs indépendants, l'activité des coursiers est fortement contrainte et encadrée par les plateformes numériques. Ce sont ces dernières qui, entre autres règles, fixent unilatéralement la tarification des courses ; celle-ci a d'ailleurs fortement varié au cours du temps à la défaveur des coursiers (voir infra la frise chronologique), les obligeant à modifier leurs manières de pratiquer l'activité pour qu'elle reste rentable. De plus, par un système de géolocalisation permettant le suivi en temps réel de leur position, elles peuvent contrôler leur activité. Enfin, elles les sanctionnent en cas de manquements réels ou supposés à différentes exigences dont la formulation est parfois floue, mais dont le non-respect peut entraîner la désactivation temporaire ou définitive de leur compte dans l'application (non-port de la tenue ou port d'une mauvaise tenue, utilisation simultanée des deux applications, comportement inapproprié avec les clients, pratique syndicale et mobilisations). Ce sont d'ailleurs ces trois caractéristiques de l'organisation du travail qui ont conduit, en France comme dans d'autres pays, à des requalifications, par décisions de justice, de ces travailleurs en salariés ${ }^{6}$. 
Les travailleurs tentent de réduire ce temps d'attente en mobilisant des tactiques pour obtenir une course plus rapidement, variables selon leur compréhension du fonctionnement - très opaque - de l'algorithme de la plateforme donneuse d'ordres. Ainsi, bon nombre de coursiers attendent au plus près des restaurants desquels émanent le plus grand nombre de commandes (essentiellement ceux de la restauration rapide), jugeant que leur géolocalisation près de ces lieux leur sera favorable. Cette stratégie largement partagée explique les rassemblements de coursiers, dès lors particulièrement apparents aux yeux des passants :

"En général quand on est à côté d'un resto on reçoit plus facilement des commandes, si on s'éloigne vraiment du centre-ville, on captera moins que les mecs qui sont plein centre, ça c'est sûr, y'a pas photo... »

Mehdi, 21 ans, licence 2 de biologie, Deliveroo, $15 \mathrm{~h} / \mathrm{sem}$.

Entretien réalisé à Poitiers, le 17/05/2019.

Photographie 2. Les coursiers attendent ensemble devant un restaurant.

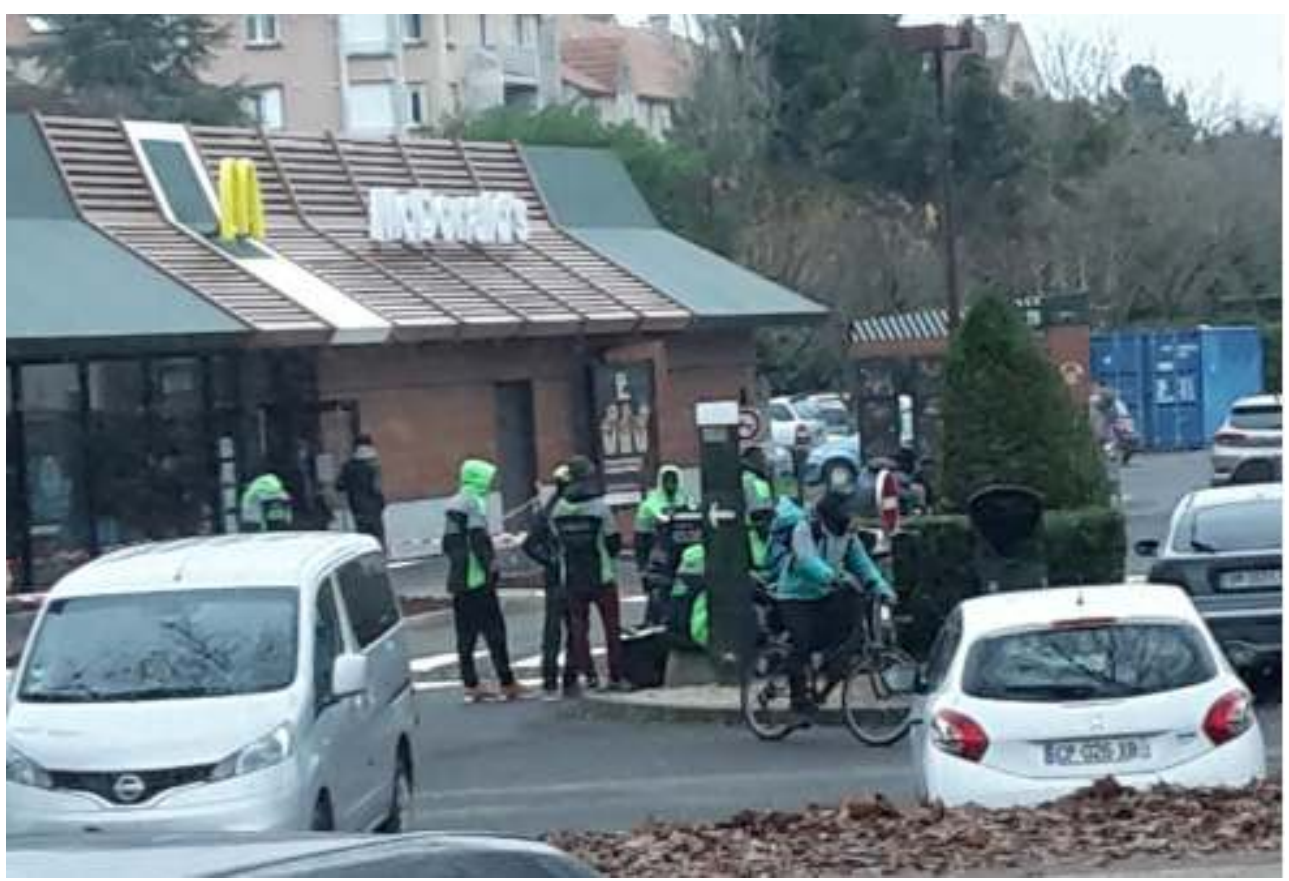

Les restaurants de restauration rapide sont ceux d'où émanent le plus de commandes de livraison. Par conséquent, leurs alentours sont investis par les coursiers qui en font des lieux d'attente. Crédit : Benjamin Néa. Poitiers, le 12 décembre 2018

6 À l'inverse, d'autres, comme Samuel, estiment que l'obtention d'une course est plus rapide lorsqu'ils sont constamment en mouvement. Si rien ne permet de prouver que cette stratégie est réellement efficace, Samuel pense que cela réactive la géolocalisation et participe à l'octroi plus rapide de courses :

"Quand des personnes n'ont pas de courses, ils se retrouvent là-bas [à côté du MacDo]... ce qui à mon avis n'est pas très stratégique parce que si on est au même endroit... ça va être un peu à plouf plouf pour celui qui a une course...

- Mais comment vous occupez les temps d'attente?

- Ben moi je fais le tour de Bordeaux...

- Toi tu roules même sans commande?

- Ouais c'est le meilleur moyen d'avoir une course c'est de rouler, d'être tout le temps en mouvement.

- Ah le mouvement ça a une importance? 
- Ouais je crois parce que quand on reste trop statique, le téléphone il peut ne pas se rafraîchir, en fait se synchroniser. Genre je passe, derrière l'opéra et je fais le tour et les mecs, je les vois dans le MacDo qui attendent et moi j'ai pas de course comme eux sauf que le fait de faire le tour de l'opéra... boum j'ai une course au MacDo où ils sont devant en fait... " Samuel, 22 ans, licence en école de commerce, Uber Eats, $\sim 21 \mathrm{~h} / \mathrm{sem}$.

Entretien réalisé à Poitiers, le 06/05/2019.

7 Pour autant, ce temps d'attente ne peut se réduire à ces tentatives de rationalisation économique car il fait l'objet de réappropriations par les coursiers. D'une part de manière isolée, en écoutant de la musique (Lemozy, 2019, 4), surfant sur internet ou s'adonnant à des jeux électroniques sur son téléphone, chacun trouve une manière de tromper l'ennui. Ceux d'entre eux qui sont par ailleurs étudiants rationalisent parfois ces moments en «travaillant pour soi » (Roy, 2006), en l'occurrence en révisant leurs cours (Tourneur, 2019, 80). D'autre part, ces temps peuvent, comme pour les chauffeurs de taxi, devenir des «moments collectifs informels propices aux interactions" (Lejeune, 2019, 2) durant lesquels ont lieu des échanges d'expériences et d'informations sur l'activité. Ils s'apparentent alors à des moments de socialisation professionnelle durant lesquels les travailleurs tentent de mieux comprendre le fonctionnement de l'application, se conseillent sur les itinéraires à prendre ou à éviter, s'informent sur les restaurateurs lents à produire les repas ou désagréables à leur égard, font le récit de leurs prouesses de livraison, se forment mutuellement aux démarches administratives à faire pour déclarer leur chiffre d'affaires, etc.

"Il n'y a pas le temps de... développer une amitié avec les collègues mais on parle de nos conditions de travail et on échange un peu là-dessus, c'est comme ça qu'on arrive à s'échanger des bons plans comme les nouveaux tarifs tout ça. "

Hugo, 24 ans, études de développeur informatique, Deliveroo, $~ 35 \mathrm{~h} / \mathrm{sem}$.

Entretien réalisé à Bordeaux, le 20/02/2019.

"Le plus souvent on se plaint! Des commandes qu'on refuse, ou sinon des restaurants qui nous font trop attendre, ça surtout, et euh... des pourboires, des gens qu'on rencontre, des clientes le plus souvent! (rires), des clients désagréables, sinon des clients fréquents atypiques... On parle de tout et de rien hein. »

Judes, 21 ans, Master Économie Finance, Deliveroo, $23 \mathrm{~h} / \mathrm{sem}$.

Entretien réalisé à Poitiers, le 05/02/2019.

Les échanges qui se nouent durant ces temps d'attente de l'activité et orientés vers elle - pouvant à ce double titre être analysés comme des temps de travail - permettent d'accroître les connaissances dont les coursiers disposent sur les stratégies possibles pour faciliter, rationaliser ou rentabiliser l'activité (voir partie 2). Tout comme la consultation et la participation à un fil de discussion entre coursiers créé sur une application numérique de messagerie instantanée, dans lequel ils échangent informations, conseils, doléances, records de courses ou de vitesse, blagues, etc. (Tourneur, 2019), ils permettent des formes de solidarité.

9 Mais ces sociabilités - et les possibilités de construction de collectifs de travail qu'elles constituent - sont précaires et fluctuantes $d u$ fait $d u$ fort taux de rotation des travailleurs. Elles sont aussi fragilisées par la concurrence inhérente à leur statut de micro-entrepreneur qui crée des tensions entre eux et limite les formes d'entraide. Les propos de Célia ci-dessous rendent compte de l'ambivalence parfois ressentie dans les relations entre les travailleurs - entre convivialité et rivalité - qui se jouent au cours de l'attente. Les coursiers, tributaires de l'algorithme qui distribue les courses d'une manière souvent pensée comme arbitraire, tentent par l'humour et le jeu d'échapper 
partiellement aux contraintes de l'attente et de désamorcer les risques de conflit interpersonnel :

"C'est quand même un peu tendu parce que quand on voit que la personne qui est là, elle a beaucoup de commandes, on est un peu... un peu jaloux quoi, enfin on se dit "pourquoi pas moi ? Pourquoi c'est pas moi qui travaille?". Donc ouais y'a un peu de tensions entre nous parfois, même moi j'ai un groupe d'Uber maintenant, je traîne avec un groupe d'Uber, avec eux, on a un point de repère au MacDo et on se pose tous au MacDo, et on a tous nos téléphones en rond comme ça et en gros on se connecte tous en même temps et on voit (rires).

- Et alors?

- Ben c'est marrant, on fait un peu de la compétition, on essaie de rendre le taf un peu marrant : qui fera plus aujourd'hui, des trucs comme ça, qui va sonner en premier, 'fin c'est plutôt marrant. »

Célia, 19 ans, $1^{\text {re }}$ année d'école de comptabilité/gestion, Uber Eats, $56 \mathrm{~h} / \mathrm{sem}$.

Entretien réalisé à Bordeaux, le 29/05/2019.

D'autres avouent plus clairement le fait qu'ils n'ont pas intérêt à partager l'ensemble de leurs «ficelles». C'est par exemple le cas de Julien qui pense avoir trouvé un bon endroit, peu connu, pour obtenir un maximum de courses et n'en dit rien à ceux qui ne sont pas ses amis :

"Moij'ai repéré cet endroit particulier...

- Comment tu l'as repéré?

- Parce que tu le vois sur la map ${ }^{7}$. Et ça m'est arrivé plusieurs fois, et du coup, quand j'ai une commande là-bas, vu que je suis pas loin, je reviens direct au resto et j'en ai une autre et parfois j'arrive à faire 10 commandes comme ça, donc c'est pas mal, je fais que dalle de courses, je gagne presque comme si je faisais des courses moyennement longues et en plus j'ai des bonus.

- Est-ce que c'est des techniques dont vous parlez entre vous?

- (Rires) Je pense qu'on évite de se dire ce genre de trucs sauf si on est vraiment potes. Mon pote avec qui j'étais en coloc à Bordeaux, il le faisait aussi donc évidemment on se partageait bien tous ces conseils mais j'irais pas le dire à un mec lambda que j'ai croisé en vélo, j'essaierais quand même de savoir ce que lui fait. " Julien, 23 ans, licence LEA Affaires et entreprises, Uber Eats et Deliveroo, $\sim 28 \mathrm{~h}$ / sem. pendant l'été.

Entretien réalisé à Poitiers, le 15/01/2019.

\subsection{Des sociabilités segmentées, des pratiques différenciées}

11 La rue est ainsi un espace de travail concurrentiel au sein duquel les temps d'attente sont aussi, comme le dit Célia, des temps de possibles tensions. Si celles-ci sont peu perceptibles au premier regard, l'est davantage en revanche la segmentation spatiale entre des groupes à la couleur de peau différente avec une polarisation entre les travailleurs noirs d'un côté et blancs de l'autre. De ce fait, les sociabilités sont également limitées parce que les coursiers ne forment pas un groupe homogène mais au contraire traversé par des segmentations.

Des proximités culturelles et le partage d'une langue commune facilitent ou au contraire freinent des rapprochements. Amadou, Guinéen, raconte ainsi qu'il a tissé des liens privilégiés avec les coursiers originaires de son pays :

"On [les Guinéens] est nombreux ici [parmi les coursiers]. On fait les dialogues, on... franchement, ça va. Parce qu'ici y'a du respect aussi, on fait les blagues comme ça, on reste assis [...]. On parle comme des travailleurs parce que on est dehors, à cause de Uber, c'est ça! Des fois, y'a des gens aussi même si on ne se connaît pas, à cause de Uber tout le monde se connait bien, travaille bien, on parlait, comme... parce que y'a beaucoup de Guinéens ici. 
Mais on n'est pas du même village. C'est à cause de Uber, parce que si y'a pas de Uber, on viendrait pas ici, ouais... "

Amadou, 25 ans, Guinéen, sans diplôme, Uber Eats, 70 h/sem.

Entretien réalisé à Poitiers, le 11/02/2019.

13 À l'instar des chauffeurs de taxi, les coursiers «forment des petits groupes dont la particularité est de se rassembler autour d'une nationalité particulière. [...] Beaucoup d'entre eux emploient leur langue natale, ce qui a pour effet de limiter la conversation entre chaque groupe" (Lejeune, 2019, 2.). Toutefois, les barrières culturelles et linguistiques n'épuisent pas la compréhension de ces sociabilités clivées. D’une part, parce qu'elles existent au sein même de ces groupes, puisque tous les travailleurs de couleur proche ne proviennent pas des mêmes pays ou régions et ne partagent pas la même langue. Mais aussi parce qu'elles ont tendance à masquer d'autres divisions déterminantes au sein des groupes ou entre eux, comme le fait d'avoir ou non la nationalité française, de posséder ou non un titre de séjour et une autorisation administrative de travailler, ou encore le fait d'être étudiant ou actif, de disposer ou non d'autres ressources financières. Ces différences entre coursiers, inscrites dans des trajectoires sociales - et pour certaines migratoires - produisent des usages différenciés de l'activité, et par là même des manières distinctes de la concevoir et la pratiquer, selon qu'ils la considèrent comme un "petit boulot", " une activité transitionnelle ", " une activité parmi d'autres", "un vrai travail», voire une « planche de salut» (Aunis, Stevens, 2020).

Parmi ces usages, une polarisation forte s'est progressivement constituée au gré du développement des plateformes de livraison de repas (Aguilera A., Dablanc L. et Rallet A., 2018). En effet, dans les premiers temps (2015-2018), cette activité était essentiellement réalisée par des jeunes, majoritairement blancs, adeptes du vélo (ils se désignent par les termes de « riders » ou «bikers ») et qui le plus souvent pratiquaient cette activité durant leurs heures disponibles à côté de leurs études ${ }^{8}$. Ils ont été progressivement rejoints par des coursiers, issus de l'immigration ou immigrés, plus particulièrement originaires d'Afrique subsaharienne, parfois en situation irrégulière, et qui, confrontés à des discriminations sur le marché du travail salarié ou sans autorisation de travail, se sont saisis de cette activité aux faibles barrières d'entrée (cf. encadré 2) pour assurer leurs revenus d'existence, la pratiquant alors à temps plein, au service de plusieurs plateformes pour maximiser les occasions de livraisons. Cette dynamique s'est accentuée et aujourd'hui, dans la plupart des villes, il semblerait que ce dernier pôle de coursiers de plateformes soit plus représenté que le premier, notamment parce que les baisses successives des tarifications et la saturation progressive du marché ont rendu l'activité moins rentable et moins attractive. Nicolas est très clair sur ce point :

«À quel moment en payant les livreurs deux euros soixante, ils [les agents des plateformes] veulent que la qualité de service reste haute? Avant, il y avait une communauté, c'était des passionnés de vélo, démographiquement tout a changé, c'est plus du tout le même truc, c'est pas du tout les mêmes profils. Franchement c'est un travail... c'est horrible ce que je vais dire mais c'est un travail d'esclave, c'est que pour les... Tous les nouveaux, c'est que pour eux, c'est un travail d'esclave pour les sans-papiers, vraiment c'est le... pour moi c'est le fin fond des chiottes du travail, désolé... »

Nicolas, 31 ans, 10 ans de restauration, Uber Eats et Deliveroo, $50 \mathrm{~h} / \mathrm{sem}$.

Entretien réalisé à Bordeaux, le 21/12/2020.

Ces différences de propriétés sociales et d'usages de l'activité, en lien avec les transformations de l'organisation qui l'affectent, produisent des divisions au sein des 
coursiers, à la fois dans les manières de la penser et de la pratiquer mais aussi dans les propensions à en accepter ou non les conditions de travail et de rémunération. C'est là que la polarisation entre coursiers de différentes origines se retrouve, par le biais de logiques de distinction, voire de disqualification sociale (Paugam, 2009). Ainsi, Judes, coursier noir d'origine guyanaise, étudiant en master, explicite ces divisions qui lui permettent de se distinguer de coursiers noirs étrangers :

"Dans notre petit comité, dans notre groupe là de bikers, on les appelle "les migrants" parce que la plupart de ces gens-là ce sont des migrants. Et c'est tout. Et en même temps, ils font Deliveroo et Uber Eats. Ils font ça TOUT LE TEMPS [en détachant les syllabes]. Tout le temps. Toujours les mêmes qu'on voit, du matin, jusqu'au soir. Ils font vraiment à temps plein. [...] Ils restent entre eux. "

Judes, 21 ans, Français guyanais, master en Économie Finance, Deliveroo, 23 h/ sem.

Entretien réalisé à Poitiers, le 05/02/2019.

De même, Sebastian, pourtant lui-même étranger de nationalité colombienne mais installé depuis presque dix ans en France et diplômé d'un master, opère cette distinction :

«Les bikers, ils sont pas tous comme ça, mais certains ils essayent vraiment de dégager un style et je pense que c'est ceux qui sont Français. [...] Alors que les étrangers, ils font pas pour le même motif. Ils s'en foutent du vélo, ils s'en foutent de... des... de tout! Ils veulent juste faire de la thune! »

Sebastian, 30 ans, diplômé d'un master 2 Création numérique, Deliveroo, $\sim 25$ h/

sem.

Entretien réalisé à Poitiers, le 13/02/2019.

17 Mathieu, quant à lui, étudiant, apparait nostalgique des sociabilités passées, constituées sur la base de groupes d'appartenance avec des habitus semblables et une passion commune pour le vélo.

"Au début tout le monde se connaissait, du coup t'arrives, si t'es pas sociable, c'est un peu compliqué...

- Les gens n'allaient pas forcément vers toi?

- Y'a un peu un jugement du nouveau "non-rider" tu vois, maintenant je sais pas si c'est pire ou si ça l'est plus du tout parce que maintenant c'est vraiment affirmé qu'il y a des gens qui sont là en vélo de ville, en jogging, qui sont pas coursiers.

- Parce qu'à l'époque c'était vraiment des mecs qui kiffaient le vélo ?

- Ouais c'était des riders... c'était des bikers, c'était trop cool, c'était une ambiance géniale.

- Et ça, ça t'a manqué au fur et à mesure du temps?

- Ouais, ouais, clairement surtout dans la convivialité en fait. Avant, enfin moi personnellement... quand t'attendais une course à un resto, que t'étais deux par exemple, ben direct tu parlais. [...] Maintenant, y'a beaucoup de... je sais pas si c'est d'immigrés mais beaucoup de gens qui parlent pas français. [...] Un jour où je logeais à l'hôtel, je me suis fait livrer Uber Eats... je me suis fait livrer un resto [...] je regarde le trajet et je vois 20 mètres... merde! Et du coup ce coursier-là, il était perdu, je le voyais il tournait, je l'appelle et impossible de se comprendre en fait,... il parlait pas un mot de français, j'essayais de lui expliquer, il comprenait pas [...]. Je suis descendu, il pleuvait, j'ai attendu 20 minutes, je lui disais : mais non c'est là, tourne à gauche... mais la place principale! C'est ta ville pourquoi tu la connais pas?"

Mathieu, 23 ans, DUT GEA, Deliveroo, 15 h à 20 h/sem. puis $8 \mathrm{~h}$ à $10 \mathrm{~h} / \mathrm{sem}$.

Entretien réalisé à Poitiers le 28/03/2019.

Les distinctions qu'opèrent ces coursiers entre les "bikers " dont ils se réclament et ceux qui "sont là en vélo de ville, en jogging, qui sont pas coursiers» recouvrent des distinctions d'origine, de nationalité et de langue (« les migrants », des «immigrés », des "gens qui parlent pas français ») qui impliquent des (stratégies de) distinctions d'ordre 
professionnel : d'une part sur les manières de penser et de réaliser le travail intensifié pour les plus économiquement dépendants à l'activité ("Ils font ça TOUT LE TEMPS »), lorsque les «bikers" peuvent l'envisager de manière plus ponctuelle et ludique ${ }^{9}$; et d'autre part sur les compétences sociales, inégalement maîtrisées, qu'il faudrait ou non posséder pour l'exercer correctement (savoir communiquer avec le client, maîtriser la géographie urbaine).

Les logiques de distinction - ou de stigmatisation - sont d'ailleurs réciproques. Certains travailleurs étrangers valorisent la force physique et la résistance à la douleur que réclame l'activité et dont ils estiment que les coursiers français seraient moins dotés. Les récits de douleurs provoquées par les longues heures passées à vélo ou les distances parcourues sont récurrents dans les entretiens. Si certains coursiers peuvent se ménager de l'effort intense en faisant des pauses plus ou moins longues, en suspendant, voire en arrêtant définitivement leur activité, les coursiers les plus dépendants économiquement doivent poursuivre malgré les douleurs. C'est le cas d'Armish, d'origine indienne, qui sous-loue un compte Deliveroo et travaille une cinquantaine d'heures par semaine malgré une blessure au genou. La résistance à la douleur se mue alors en compétence nécessaire aux yeux de ces travailleurs. Ainsi Koffi, Ivoirien sans papier de 32 ans considère que ce travail n'est pas fait pour " pour quelqu'un qui ne veut pas accepter la douleur » et qu'il « faut des gens solides »; il retourne alors le stigmate dont il est l'objet (Goffman, 1975) pour disqualifier à son tour des coursiers français qui ont arrêté et retirer une forme de gratification symbolique à disposer de ressources telles que le « courage » et la « détermination » qui lui permettent d'exercer ce travail.

Cette polarisation peut dans les faits être plus nuancée, du fait de variations internes causées de la même façon par les situations sociales et économiques de chacun, plutôt que par les nationalités des travailleurs stricto sensu. Par exemple Aloïs, qui considère que "quand il fait beau, c'est le plus beau taf du monde» (cf. infra), estime travailler 48 heures par semaine. Inversement, Ibrahim, travailleur marocain qui vient juste d'obtenir en 2019 un titre de séjour après dix années de présence illégale en France, dont plusieurs sans domicile fixe, travaille à temps partiel comme coursier pour Uber Eats. En effet, malgré sa précarité économique, il doit préserver du temps pour s'occuper de sa fille de six ans dont il a la garde partagée avec son ex-compagne. Enfin, les divisions peuvent être en partie dépassées à l'occasion de mobilisations (cf. photographie 7, partie 3) qui peuvent rassembler des travailleurs jusqu'alors éloignés les uns des autres.

21 Mais il n'en demeure pas moins que ces lignes de partage dessinent malgré tout des logiques sociales différenciées d'accès et de pratiques de l'activité de coursiers, logiques qui se reconstituent spatialement par groupes d'affinités lors des temps d'attente. Le fait que ces temps se déroulent en grande partie sur les places - espaces urbains entourés de restaurants, lieux de convergence et de divergence par les rues qu'elles distribuent, endroits propices aux rendez-vous, aux rencontres informelles comme aux rassemblements - rend la présence des coursiers dans la ville particulièrement visible. Investis par les travailleurs comme un moyen de se retrouver, ces temps improductifs sont alors l'occasion de sociabilités professionnelles et de développement de petites stratégies d'optimisation, invisibles au regard des passants mais pourtant déterminantes pour les coursiers. 


\section{Entraperçus dans les rues : optimiser (le temps de) la course}

Malgré leurs tactiques, les coursiers ne peuvent écourter de manière significative les deux «temps morts»: attente de l'attribution de la commande, attente de la préparation du repas. Les seuls moments sur lesquels les livreurs peuvent véritablement agir sont ceux des trajets, durant lesquels ils se rendent au restaurant pour récupérer la commande attribuée, puis la livrent du restaurant au client. Parmi ces deux trajets, seul le dernier, considéré comme la course proprement dite, est rémunéré.

\subsection{Rentabiliser l'activité, foncer et prendre des risques}

L'enjeu de ce travail payé à la tâche est dès lors de le rendre le plus productif et rémunérateur possible, en jouant sur sa vitesse d'exécution dès réception de la commande (Lemozy, 2019). C'est ce que raconte Sebastian qui a rationalisé ses gestes au point de les mimer en entretien tel un automate :

«Je rentre [dans le restaurant], tchouc, je sors mon sac, je l'ouvre déjà pour pas l'ouvrir après quand on me donne la commande, je le laisse près pour que quand la commande soit prête, BAM! Poser le truc, fermer, tchouc tchouc tchouc, et j'me tire. Donc même dans les petits détails je suis déjà en train d'essayer de gagner du temps. Gagner du temps... gagner du temps... [...] Je récupère la commande, je pars le plus vite vers le client, l'adresse, et quand je suis sûr que le client ben il habite là, qu'il m'a vu, qu'il sait que je suis là et que j'arrive, je valide la commande même sans voir sa tête ou rien, c'est TOUT dans la vitesse. Et tout de suite, si j'ai d'la chance, ben... je reçois une nouvelle commande que j'peux déjà accepter [...]. $J$ 'essaye de tout faire super rapidement, $t t-t t-t t-t t$ [bruit avec la langue pour marquer la cadence], d'enchaîner enchaîner. "

Sebastian, 30 ans, diplômé d'un master 2 Création numérique, Deliveroo, $\sim 25$ h/ sem.

Entretien réalisé à Poitiers, le 13/02/2019. 
Photographie 3. La course en actes (1). Foncer ou chercher son itinéraire.

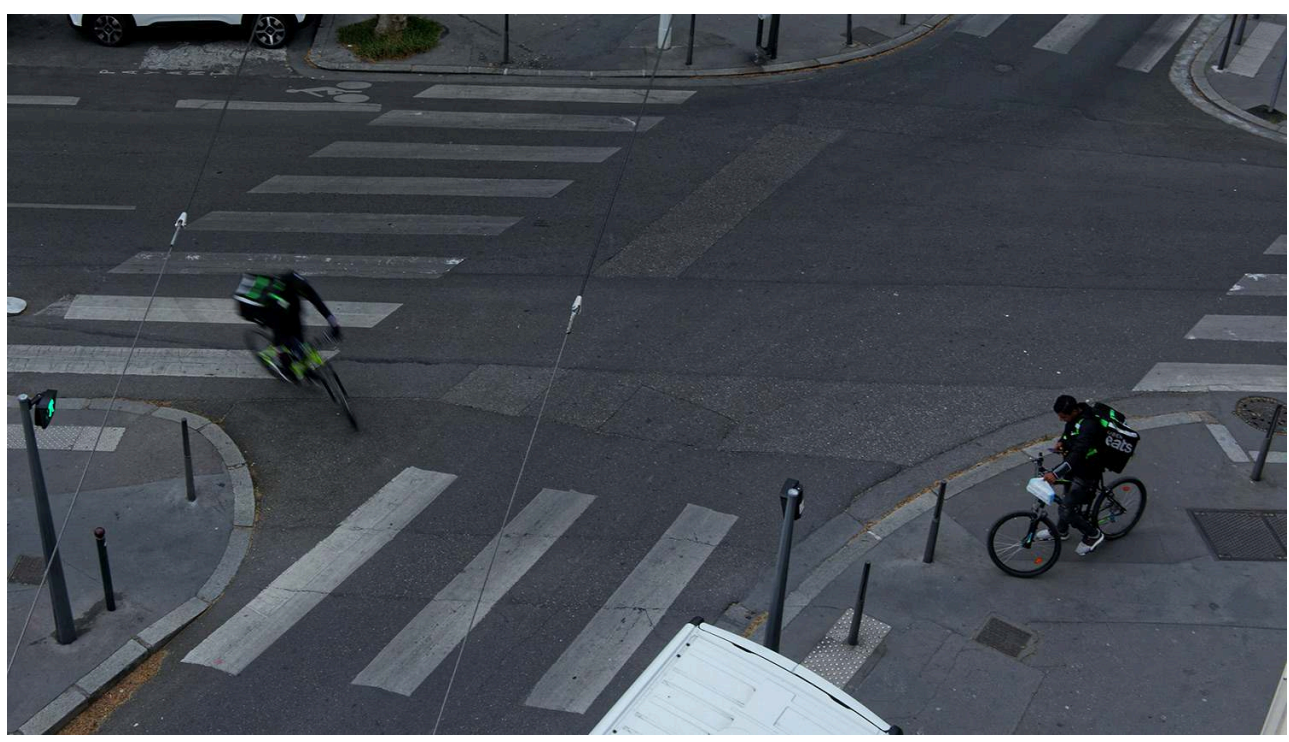

La photographie 3 met en évidence différentes tâches qui constituent l'activité de livraison. Tandis que le coursier situé à droite de l'image semble chercher son itinéraire à partir de son téléphone, la partie gauche de l'image met en exergue le mouvement, il faut foncer pour être rentable.

Crédit : Henri Eckert, Lyon, 15 avril 2020.

Durant le déplacement en particulier, il s'agit d'être le plus rapide possible, en sollicitant sa force physique et son endurance pour pédaler vite et longtemps, en recherchant tout ce qui permet une accélération (raccourcis compris) et en évitant tout ce qui pourrait ralentir ou freiner la cadence (sens interdits, feux rouges, stop, ralentissements de la circulation, zones piétonnières aux horaires d'affluence, dénivelés trop importants, etc.).

Ces pratiques d'autoaccélération sont accentuées par des systèmes de classements des meilleurs coursiers ou de primes allouées par les plateformes en échange d'un nombre de livraisons effectuées en un temps restreint et souvent difficile à atteindre. Elles sont aussi soutenues par un sens du travail bien fait, qui vise la satisfaction du client à qui le repas est livré chaud et rapidement. Ainsi Hugo précise :

«Il n'y a personne de la hiérarchie qui est là pour nous dire il faut que tu accélères... donc du coup le fait d'apporter la nourriture chaude, c'est juste une contrainte qu'on se met à soimême. OK, il y a le chiffre à l'arrivée, oui c'est ça qui nous booste, c'est ça qui nous motive, mais moi je sais que psychologiquement c'est ça qui nous motive mais aussi le fait d'apporter un repas chaud quand même au client parce que... faut quand même y penser un minimum, c'est pour eux qu'on rend le service, on est payés nous mais c'est quand même à la base pour le client. "

Hugo, 24 ans, études de développeur informatique, Deliveroo, $35 \mathrm{~h} / \mathrm{sem}$.

Entretien réalisé à Bordeaux, le 20/02/2019. 
Photographie 4. La course en actes (2). Foncer et prendre des risques.

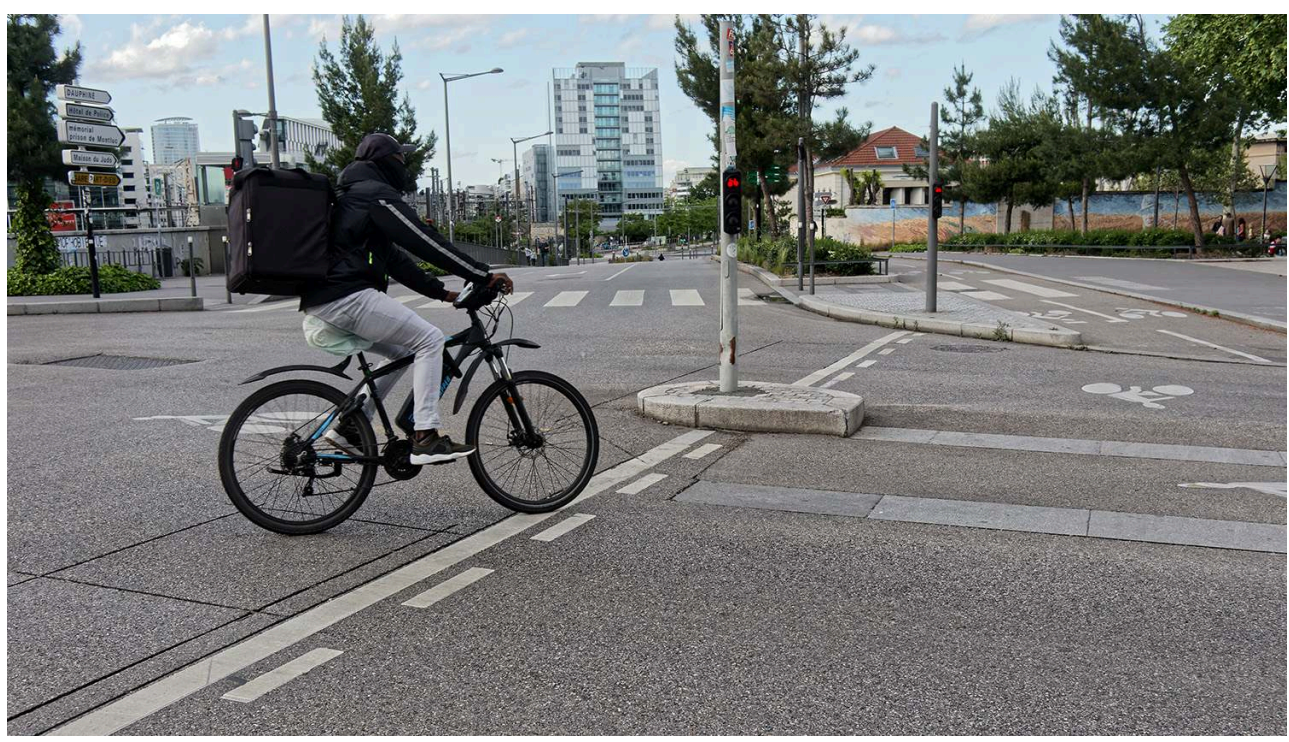

La photographie 4 montre un coursier qui, profitant d'une faible circulation, prend un carrefour en sens interdit afin d'optimiser son trajet.

Crédit : Henri Eckert, Lyon, 30 avril 2020.

C'est ainsi que l'on peut voir les coursiers foncer dans les rues, mettre leur endurance physique à l'épreuve, commettre des infractions routières et risquer les accidents (photo 4). Ces pratiques expliquent d'ailleurs le fait que les coursiers sont presque exclusivement des hommes : non seulement le vélo est, statistiquement, plus pratiqué par les garçons et les hommes que par les filles et les femmes ${ }^{10}$, les prédisposant davantage à l'activité de coursier, mais la manière de le pratiquer diffère selon le genre : alors que les femmes ont plus fréquemment intériorisé l'impératif de sécurité, les hommes ont une pratique plus diversifiée dans les formes et lieux de pratiques (vélo de course, de ville, VTT, BMX, fixie) ${ }^{11}$, plus ludique, plus affranchie du code de la route et des règles de sécurité, et marquée par la compétition et le défi ${ }^{12}$. En vélo comme dans bien d'autres activités, la prise de risque mais aussi la résistance à l'effort ou à la souffrance, la recherche de la performance, appartiennent au registre de la masculinité. Or, le travail de coursier fait précisément appel à cet usage de confrontations virilistes du vélo, de surcroît dans une ville «faite par et pour les hommes » (Raibaud 2015).

Si l'engagement corporel des coursiers est visible, l'est à l'inverse moins toute une série d'opérations cognitives pour élaborer en permanence des stratégies visant à ménager ou réduire sa peine tout en ayant un maximum de courses. La première d'entre elles consiste à estimer rapidement, au moment de l'attribution d'une commande, si le rapport entre le coût en termes de temps et d'effort fournis pour la course et la rémunération à laquelle elle donnera lieu sera favorable (ou le moins défavorable) afin de décider s'il est préférable de l'accepter ou de la refuser. Mais la fragilité des informations disponibles et la variabilité des modes de tarification dans le temps (voir frise chronologique ci-dessous) rendent le calcul incertain, comme l'explique Sebastian :

"J'essaye d'analyser quelle course va me prendre tel pourcentage de temps et est-ce que c'est une bonne affaire ou est-ce que c'est une bonne idée d'annuler et attendre d'autres commandes qui peuvent être plus intéressantes, mais tu sais jamais parce que tu sais pas 
quand est-ce que les gens vont commander mais bon. À peu près on sait. »

Sebastian, 30 ans, diplômé d'un master 2 Création numérique, Deliveroo, $\sim 25 \mathrm{~h}$ /

sem.

Entretien réalisé à Poitiers, le 13/02/2019.

Frise chronologique des modifications tarifaires des plateformes Uber Eats et Deliveroo en France.

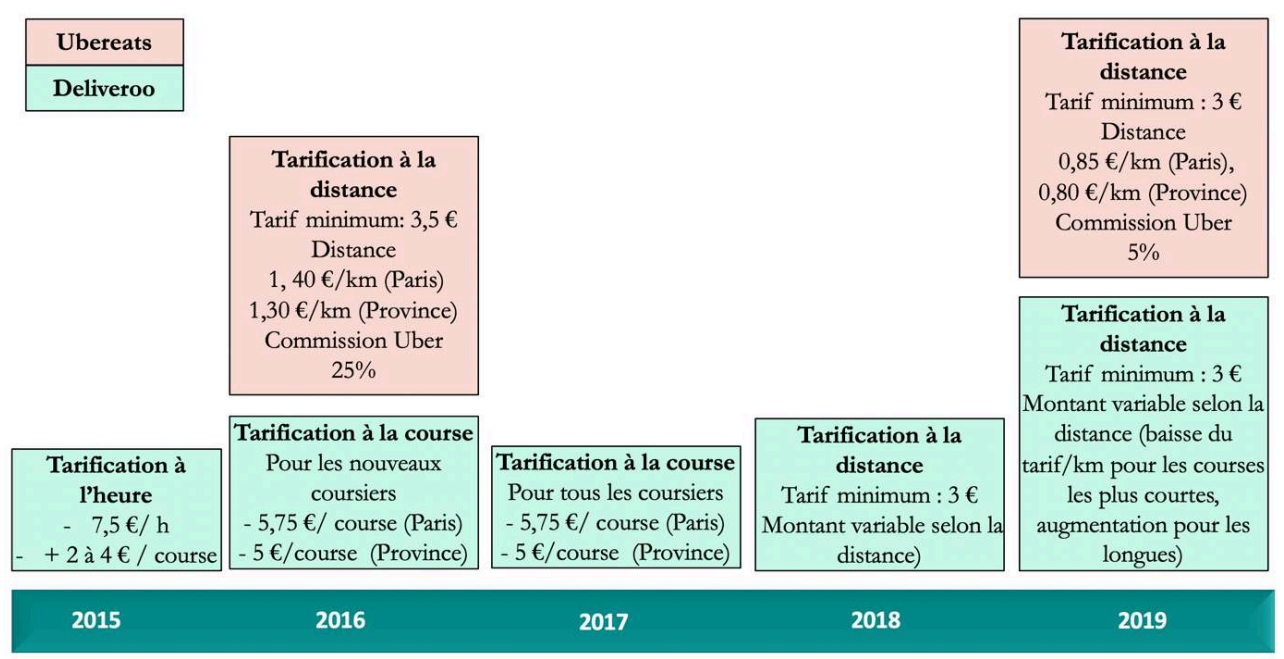

Source : autrices, à partir des entretiens, d'articles de presse et de sites de plateformes.

d'une course. Celle racontée par Hugo est courante :

"Un euro la réception, deux euros la livraison, un euro trente par kilomètre, le problème c'est que ça prend en compte que du restaurant jusqu'à chez le client donc... D'ailleurs ça a donné lieu à une très mauvaise expérience que j'ai eue où j'étais au nord de la ville et en fait j'ai été appelé pour un restaurant qui était à la limite du boulevard mais quasiment au sud... donc j'ai dû traverser tous les boulevards, jusqu'au sud donc vraiment c'était assez long, et je récupère la pizza et je vois que la livraison doit se faire à 600 mètres, genre au bout de la rue $d u$ resto, donc j'y vais tranquillement, j'arrive devant le client, je lui donne sa commande, je valide et là je regarde ce que j'ai reçu, j'avais reçu 4 euros 60 ... la commande était vraiment tout petite alors que moi j'avais euh... bien 5-6 kilomètres derrière moi. »

Hugo, 24 ans, études de développeur informatique, Deliveroo, $~ 35$ h/semaine.

Entretien réalisé à Bordeaux, le 20/02/2019.

Chaque coursier élabore, par l'expérience et les échanges avec ses pairs, sa propre stratégie, en lien avec les règles de tarification des plateformes et du chiffre d'affaires qu'il souhaite dégager. Samuel préfère accepter les courses de courte distance, sauf en cas d'affluence de coursiers :

"C'est toujours mieux d'avoir des courses courtes parce que du coup on enchaîne plus vite et même si on ne se fait pas beaucoup d'argent sur la course parce que c'est aléatoire, on aura toujours plus de courses, du coup on pourra accéder à des primes parce que les primes se mettent en fonction des courses réalisées. Par contre dans une soirée où il y a beaucoup beaucoup de monde, il n'y aura pas forcément de petites courses et il faudra préférer les grandes courses comme ça on s'éloigne mais le temps qu'on s'éloigne, on arrive à attraper une autre course même si elle est [lointaine], au moins on en a une et c'est mieux payé. » Samuel, 22 ans, licence en école de commerce, Uber Eats, $21 \mathrm{~h} /$ semaine.

Entretien réalisé à Poitiers, le 06/05/2019. l'investissement dans un matériel permettant cette optimisation. Une manière de répondre aux impératifs d'autoaccélération avec des règles floues tout en préservant sa 
santé est ainsi de s'équiper d'un vélo à assistance électrique ou même d'un scooter, ce qui crée des distinctions entre travailleurs comme l'explique Mehdi qui possède un vélo ordinaire :

«Après ça dépend de chacun aussi, si on se décide de vouloir prendre que la commande qui va être à 200 mètres du resto, ben tu vas faire 4 euros 50 dans ta soirée, si tu te décides de faire les commandes acceptables quand t'as pas un vélo électrique c'est-à-dire les commandes où c'est pas trop de montées, même s'il y a un peu de distance moi ça me dérange pas [...] En général moi j'accepte uniquement les commandes vraiment centre-ville. Parce que c'est du plat même si c'est un peu loin et j'accepte aussi toutes les commandes $d u$ côté gauche du centre-ville [...] et pas du côté droit, parce que je remonte systématiquement par les ascenseurs de la gare [située sur le côté gauche de la ville]. Là il y a deux ascenseurs qui sont quasiment jamais utilisés et on a la place pour mettre notre vélo et on arrive en haut de la passerelle, on n'a plus qu'à se taper la petite montée [...]. Moi je l'utilise uniquement parce que j'ai un vélo mécanique, un gars qui est à l'électrique c'est pas bénef de faire ça, autant qu'il monte toute la montée à 25 à l'heure il ira plus vite que moi. » Mehdi, 21 ans, licence 2 de biologie, Deliveroo, 15 h/sem.

Entretien réalisé à Poitiers, le 17/05/2019.

31 On le voit avec Mehdi qui ne prend que les livraisons sans dénivelés : décider d'accepter ou refuser une commande nécessite également de bien connaître la ville afin d'évaluer la pénibilité du parcours. Édouard, moins familier que Mehdi de la topographie urbaine, en a fait les frais :

«Tu peux te retrouver face à des merdes tu vois. Genre t'as mal calculé la distance d'un truc, toi tu pensais que c'était plus près ou tu avais pas anticipé une énorme montée. J'me suis déjà tapé un truc jusqu'à la caserne militaire, sachant que je savais pas qu'il y avait une grande montée pour y aller, mais j'connaissais pas trop les environs non plus. »

Édouard, 24 ans, $3^{\mathrm{e}}$ cycle Conservatoire Théâtre, Deliveroo, $~ 8 \mathrm{~h} / \mathrm{sem}$.

Entretien réalisé à Poitiers, le 20/12/2018.

Cette connaissance empirique, issue de l'expérience et des échanges entre pairs, soutient les pratiques d'autoaccélération, parce qu'elle permet de ne pas hésiter sur l'itinéraire, de ne pas avoir à faire demi-tour, de prendre des raccourcis, etc. Elle donne dans le même temps aux coursiers un sentiment de réappropriation du travail parce qu'elle leur permet de s'affranchir du système de guidage de l'application, comme l'indique Célia, mais aussi de le manipuler à son profit, comme le raconte Julien :

«Le GPS parfois, les sens interdits, les feux, même euh... je sais pas des fois j'ai l'impression qu'il donne le chemin le plus long pour faire plus de kilomètres parce que des fois je me dis mais pourquoi je passerais par là alors que je pourrais passer par là. »

Célia, 19 ans, $1^{\text {re }}$ année d'école de comptabilité/gestion, Uber Eats, 56 h/sem.

Entretien réalisé à Bordeaux, le 29/05/2019.

"L'appli elle est con, en fait elle te fait aller dans le sens de circulation et du coup, tu es sur les quais, tu as le MacDo qui est là, tu as la rue qui est là et généralement tu fais des commandes qui sont dans la rue ici, là, sauf que l'appli elle te fait faire 36000 détours et elle te paye beaucoup plus alors que toi tout ce que tu as fait, c'est 200 mètres comme ça... Donc tu es payé comme si tu avais fait 2 kilomètres alors qu'en fait tu as fait 500 mètres. " Julien, 23 ans, licence LEA, Uber Eats et Deliveroo, 28 h/sem. pendant l'été. Entretien réalisé à Poitiers, le 15/01/2019.

Ainsi, l'activité des coursiers, comme celle d'autres travailleurs d'univers de travail très prescrits et contraints, implique des formes de rationalité et mobilise des compétences largement invisibles et méconnues. Et l'on y saisit le même refus de s'en remettre totalement à l'application conçue pour encadrer et contrôler leur travail et imposer gestes et cadences, refus de ne pas se soumettre à cette machine algorithmique, de ne pas y être associé (Gaborieau, 2017), mais de chercher constamment, dans ses failles, les 
marges de manœuvre qui permettent d'affirmer son libre-arbitre, de garder une prise, aussi minime soit-elle, sur le travail, et mieux, de se le réapproprier ${ }^{13}$.

Mais ces formes de rationalité et ces savoirs sont fragiles car ils sont extrêmement dépendants de l'organisation formelle définie par les plateformes, et de leur algorithme au fonctionnement très opaque et régulièrement modifié. Les changements arbitraires des règles du jeu (et des rémunérations) par les plateformes remettent alors en cause tous les petits aménagements pour rendre le travail à la fois rentable économiquement, supportable physiquement et acceptable moralement. Dépossédés de leurs savoirs, les coursiers travailleurs n'ont souvent d'autre choix que de se soumettre au dispositif technique et organisationnel (Linhart, 2015) ${ }^{14}$.

\subsection{Des stratégies précaires et différenciées}

Limitées et fragiles, ces logiques d'intensification de l'activité et les stratégies associées pour la rendre tenable sont également variables. Elles ne se jouent en effet pas de manière identique pour tous les coursiers et s'apparentent à une palette dans un continuum entre les deux pôles définis dans la première partie: d'un côté, ceux qui pratiquaient le vélo avant de se saisir de l'activité de coursier comme d'une occasion de concilier loisirs et travail rémunéré (les " bikers »). Moins dépendants économiquement de l'activité, ils peuvent se ménager en l'exerçant à leur gré, quelques heures par jour et quelques jours par semaine, mais également en s'autorisant davantage à refuser des commandes jugées trop lointaines ou pénibles ${ }^{15}$. Pour rendre plus soutenable mais aussi plus attrayante leur activité, ils ont tendance à investir dans des vélos légers, de course ou fixie, et cherchent dans l'accélération un plaisir et un challenge sportifs. Ainsi, ils introduisent du jeu dans le travail (Roy, 2006 ; Savignac, 2017), en tentant de battre des records de vitesse (les leurs, ceux de leurs pairs et même ceux de sportifs amateurs ou professionnels) ou de distance ou dénivelé parcourus dans la journée, en organisant entre eux des concours de livraison, ou en faisant la course contre des voitures. Ils partagent leurs exploits lors des temps d'attente partagés ou sur leur fil de discussion numérique. Le terme de course prend alors le double sens de livraison («faire une course ») et de compétition (" faire la course ») :

"Cette semaine j'ai fait des grosses distances de dénivelé tout ça et donc c'est... moi je sais que sur Strava, sur l'application Strava, la géolocalisation machin, je peux comparer des temps à des professionnels et je suis carrément dans les cordes alors qu'au final je suis pas un cycliste comme je disais tout à l'heure, ça m'arrive de faire des sorties mais mon activité principale c'est vraiment la livraison de plats chauds avec Deliveroo, et c'est hallucinant de voir que par le biais d'un travail il y un challenge sportif derrière qui ressort et qui peut être à la hauteur pas des meilleurs mais de bien d'autres cyclistes, qui font vraiment ça pour l'activité sportive."

Aloïs, 22 ans, bac éco ES, trois mois en fac éco, Deliveroo et Uber Eats, $\sim 48 \mathrm{~h} / \mathrm{sem}$. Entretien réalisé à Poitiers, le 06/05/2019.

Alors que l'activité est socialement dévaluée et économiquement précaire, ces coursiers peuvent la maintenir à distance en l'exerçant à temps partiel et en y trouvant des sources de reconnaissance par les pairs et de plaisir :

"Je roule... vite. Vraiment vite. J'aime ça. Dans une descente, je peux pas rouler à moins de cinquante. J'aime trop aller vite. "

Sebastian, 30 ans, diplômé d'un master 2 Création numérique, Deliveroo, $\sim 25$ h/

sem.

Entretien réalisé à Poitiers, le 13/02/2019. 
"Quand il fait beau c'est le plus beau taf du monde et je pense même pas à la rémunération, je pense même pas à nos conditions de..., je ne pense même pas à tout ce que la société englobe autour du taf, au final c'est vraiment juste de la vie pure et simple, t'es sur ton vélo, tu gagnes ton argent sur ton vélo, tu vas vite si tu veux, tu vas lentement si tu veux... il y a énormément de liberté, on a quand même, malgré tout, énormément de liberté. »

Aloïs, 22 ans, bac éco ES, trois mois fac éco, Deliveroo et Uber Eats, 48 h/sem.

Entretien réalisé à Poitiers, le 06/05/2019

D'un autre côté, se trouvent des coursiers qui le sont devenus pour accéder à un revenu et qui, pour que celui-ci atteigne un niveau souhaité ${ }^{16}$, travaillent de longues heures quotidiennement, en dépit du temps extérieur ( $a$ fortiori les jours pluvieux ou neigeux $\mathrm{du}$ fait des primes proposées) et acceptent les courses quelles qu'en soient la distance et les pénibilités. Pour tenir dans ce travail d'autant plus intensifié et endurant - mais aussi pour le rendre plus productif, ils investissent plutôt dans des vélos à assistance électrique ou dans des scooters. C'est d'ailleurs ce qu'observe Mehdi :

\footnotetext{
"Il y a des mecs qui acceptent tout mais c'est des gars qui ont des vélos électriques et qui travaillent toute la journée, ils ont deux batteries de côté dans leur appartement et ça leur change strictement rien d'aller à [nom d'une commune limitrophe] ou en bas du [lieu proche du centre-ville]. Au contraire, ils sont payés un euro de plus pour remonter! Moi, avec un vélo mécanique, si on me propose une commande à 4 euros 50 pour rester en centreville ou à 6 euros 50 pour monter à [nom de la commune limitrophe], je prends celle à 4 euros 50 en centre-ville, c'est logique, mais du coup mon taux d'acceptation doit être moindre. »

Mehdi, 21 ans, licence 2 de biologie, Deliveroo, $\sim 15 \mathrm{~h} / \mathrm{sem}$.

Entretien réalisé à Poitiers, le 17/05/2019.
}

Si on les voit foncer dans les rues, un ensemble de compétences ou stratégies reste invisible "à l'œil nu»: le choix des itinéraires, les pratiques d'intensification ou d'aménagement de l'effort, l'équipement choisi qui constituent autant de manières de travailler relatives aux usages de l'activité et aux profils sociaux des coursiers. Ces compétences invisibles vont aussi de pair avec des injonctions à se rendre invisibles, qui se voient parfois renversées lors des mobilisations.

\section{Passer inaperçu ? Entre injonctions à la discrétion et mobilisations dans l'espace public}

\section{1. (Devoir) se faire invisible}

Les coursiers sont donc visibles dans l'espace public même si une partie des caractéristiques de l'activité et des stratégies qu'ils développent reste imperceptible. Leur présence aux yeux de tous a en corollaire rendu manifeste la progressive transformation de la population des livreurs, caractérisée par le passage d'un groupe majoritairement composé de livreurs blancs, étudiants, exerçant l'activité à temps partiel à celui de livreurs étrangers ou d'origine étrangère, exerçant le plus souvent à temps plein.

Cette présence, qui s'est progressivement massifiée au fil du temps, est dès lors devenue dérangeante pour les plateformes et certains restaurateurs. Très mal rémunérés, sans couverture sociale face aux risques du travail, ils sont très vite devenus dans les médias et une partie de l'opinion publique, le symbole malheureux de l'économie de plateforme. Leur visibilité, rappel du stigmate de leur exploitation, a pu alors gêner ${ }^{17}$. 
Photographie 5. Des coursiers postés devant le restaurant Quick.

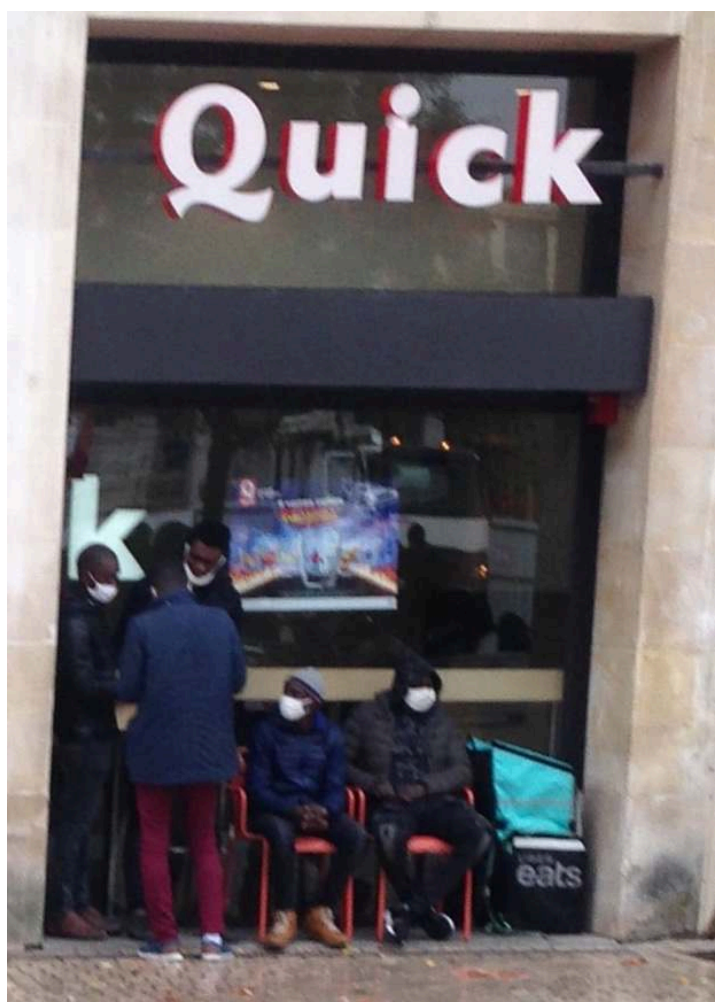

Un jour pluvieux, les coursiers attendent les commandes et se sont abrités contre la devanture du restaurant et installés sur des chaises inutilisées par les clients.

Crédit : Hélène Stevens. Poitiers. 2 novembre 2020.

La société Uber Eats a organisé en mars 2019 une consultation auprès de ses livreurs ${ }^{18}$ qui intègre les critiques de riverains, commerçants, restaurateurs ou municipalités et cherche à intervenir pour réguler leur présence ostensible. C'est ce qu'explique une des cadres de l'entreprise, stigmatisant les comportements d'incivilité de certains :

"Les nuisances, il y a plusieurs cas évidemment, il y a tout simplement le fait qu'il y a plusieurs coursiers qui vont attendre devant un restaurant juste en bas de chez vous, ça fait du bruit, ils sont sur le trottoir, ça peut gêner des gens... Et après il y a des comportements qui ne sont pas de notre ressort, qui ne sont pas liés à l'activité qu'on propose, qui sont juste des comportements d'incivilité de certains coursiers et le fait qu'ils aient un sac à dos Uber Eats ou Deliveroo, ça peut être... c'est pas du tout lié à l'activité, du coup on est un peu pris à partie pour un coursier qui va griller un feu rouge, qui va... je sais pas... avoir un comportement illégal, incivil, et ça c'est un peu difficile pour nous [...] mais après sur des éléments plus d'attroupements, on essaie en partenariat avec les restaurants ou avec les mairies d'installer du mobilier urbain par exemple où les coursiers vont pouvoir s'asseoir en attendant une commande, vont pouvoir recharger leurs téléphones portables, vont pouvoir garer leurs vélos. »

Christelle, cadre salariée d'Uber Eats, entretien réalisé à distance, le 30/07/2020.

Sans que l'on sache s'il s'agit d'une consigne venant des plateformes avec lesquelles ils ont noué un partenariat ou d'une initiative de leur part, certains restaurateurs cherchent à masquer la présence de leurs livreurs aux abords de leur entreprise en leur interdisant de se poster directement à l'entrée :

"Je vais au MacDo où il y a beaucoup de monde qui y va et du coup on a été virés, on n'a plus le droit d'attendre aux alentours du MacDo [...] parce qu'apparemment les gens se sont 
plaints. C'est clair qu'il y avait un bel attroupement de personnes mais du coup on n'a plus le droit d'y aller donc on se met aux alentours parce qu'il y a pas mal de fast-foods.

- Et comment vous l'avez su que vous n'aviez plus le droit d'y aller?

- Ben on a reçu un message, un SMS. "

Boris, 21 ans, L3 STAPS, Uber Eats, $5 \mathrm{~h} / \mathrm{sem}$.

Entretien réalisé à Poitiers, le 06/05/2019.

Alors qu'ils sont exposés aux yeux de tous puisque travaillant dans la rue, les coursiers sont ainsi enjoints à se rendre invisibles. Et, pour la même raison, ils ne peuvent pas non plus se réfugier dans les restaurants lorsqu'ils attendent leur commande, ce que déplore Samuel :

«Parfois il y a des restaurants où on est considérés un peu comme les toutous qui vont chercher voilà. Où par exemple quand il fait limite zéro on nous dit si on peut attendre dehors alors qu'il y a deux personnes dans le resto. En fait, ils prennent l'habitude de nous dire "Attendez dehors etc." alors qu'on gêne personne, même si on est au niveau de la porte ça gêne personne qu'on soit là ».

Samuel, 22 ans, licence école de commerce, Uber Eats, $\sim 21 \mathrm{~h} / \mathrm{sem}$.

Entretien réalisé à Poitiers, le 06/05/2019.

Ces injonctions relèvent d'une logique sociale selon laquelle la visibilité du travailleur s'accompagne d'une volonté d'invisibilisation du processus de travail (Krinsky, Simonet, 2012). Savoir se faire oublier n'est pas une caractéristique exclusive du travail des coursiers, elle est plus largement le fait des activités de services peu valorisées et qui doivent passer inaperçues. Il en va ainsi des éboueurs, "maitres de force invisibles » (Corteel, Le Lay, 2011, 26), des travailleuses du care qui doivent faire en sorte que celles et ceux qui bénéficient de leur travail « ne sa[chent] pas ce qu'il en a coûté à la personne qui a produit le service » (Molinier, 2010,165) ou des caissières qui doivent se faire discrètes (Bernard, 2013). Comme pour ces travailleurs et travailleuses, l'un des aspects de l'activité de coursier consiste à se rendre invisible, comme si leur seule présence pouvait déranger, rappelant l'existence des hiérarchies sociales et des métiers.

En d'autres termes, s'ils sont apercevables car exerçant dans la rue, ils doivent passer inaperçus, ce qui est intériorisé par une partie des travailleurs eux-mêmes. En effet, les coursiers, à l'instar des caissières, considèrent la discrétion comme caractéristique du travail bien fait, «invisibiliser son travail» devient «une question de conscience professionnelle» (Bernard, 2013, 128). C'est le cas de Mehdi qui, par ses usages de l'espace public, cherche à ne pas déranger : afin de se ménager, il utilise parfois un ascenseur public situé près du théâtre et d'un restaurant partenaire de sa plateforme pour échapper à un dénivelé (cf. partie 2). Toutefois, il n'utilise ce moyen que le dimanche, lorsque théâtre et restaurant sont fermés :

«Moi je le fais pas en semaine, je le fais pas en semaine parce que je pense que ça finirait par les embêter un peu, même si on aurait le droit mais on sait très bien que les restaurateurs ils aiment bien leur territoire, leur clientèle qu'elle soit pas dérangée même si ça dérange pas forcément mais le dimanche vu que c'est fermé [...] je prends l'ascenseur et puis bon je suis déjà en centre-ville. » Mehdi, 21 ans, licence 2 de biologie, Deliveroo, 15 h/sem. Entretien réalisé à Poitiers, le 17/05/2019

Cette exigence devient même un impératif lorsque les coursiers sont sans-papiers et que leur présence dans l'espace public constitue pour eux un risque permanent de confrontation aux autorités publiques et une menace d'arrestation. 


\section{1. (Vouloir) se rendre visibles}

47 Mais ces tentatives d'invisibilisation, qu'elles proviennent des restaurateurs, des plateformes, voire des travailleurs eux-mêmes, ne sont pas garanties de succès. En témoignent au contraire les mobilisations répétées des coursiers, particulièrement nombreuses et médiatisées, pour contester les conditions de rémunération imposées et constamment redéfinies par les plateformes. Ces mobilisations revêtent d'ailleurs un caractère régulier, puisqu'elles ont eu lieu durant les étés 2017, 2018 et 2019, à chaque fois suite aux modifications tarifaires unilatéralement décidées par les plateformes ${ }^{19}$. Elles sont l'occasion de prises de vue par les coursiers, à la fois pour « immortaliser » un collectif de travailleurs mais aussi rendre compte de la contribution d'un collectif local de revendications à une mobilisation nationale et l'afficher sur les réseaux sociaux numériques. On trouve alors sur ces réseaux de nombreux clichés, résultats d'une mise en scène rappelant sous certains aspects une désormais longue tradition de photographies du travail usinier (Assegond, 2012) et d'occupation d'usine par les ouvriers et ouvrières (Nerrière, 2016) : compositions parfois étagées (photographie 6) de travailleurs posant avec leur outil de travail placé de manière ostentatoire devant eux ; certains sont de face, les regards convergeant vers le photographe tandis que d'autres choisissent de garder l'anonymat en tournant le dos (photographie 6) ou en portant des masques de déguisement (photographie 7); brandissant qui une veste achetée à la plateforme de livraison pour laquelle il travaille (photographie 6), qui une banderole ou des pancartes (photographie 7); faisant le «V de la victoire» (photographie 6) ou brandissant le poing (photographies 7 et 8), autant d'adresses aux plateformes numériques et aux pouvoirs publics et de signes pour faire part de leur lutte collective. 
Photographie 6. Mobilisation de coursiers nantais en août 2019.

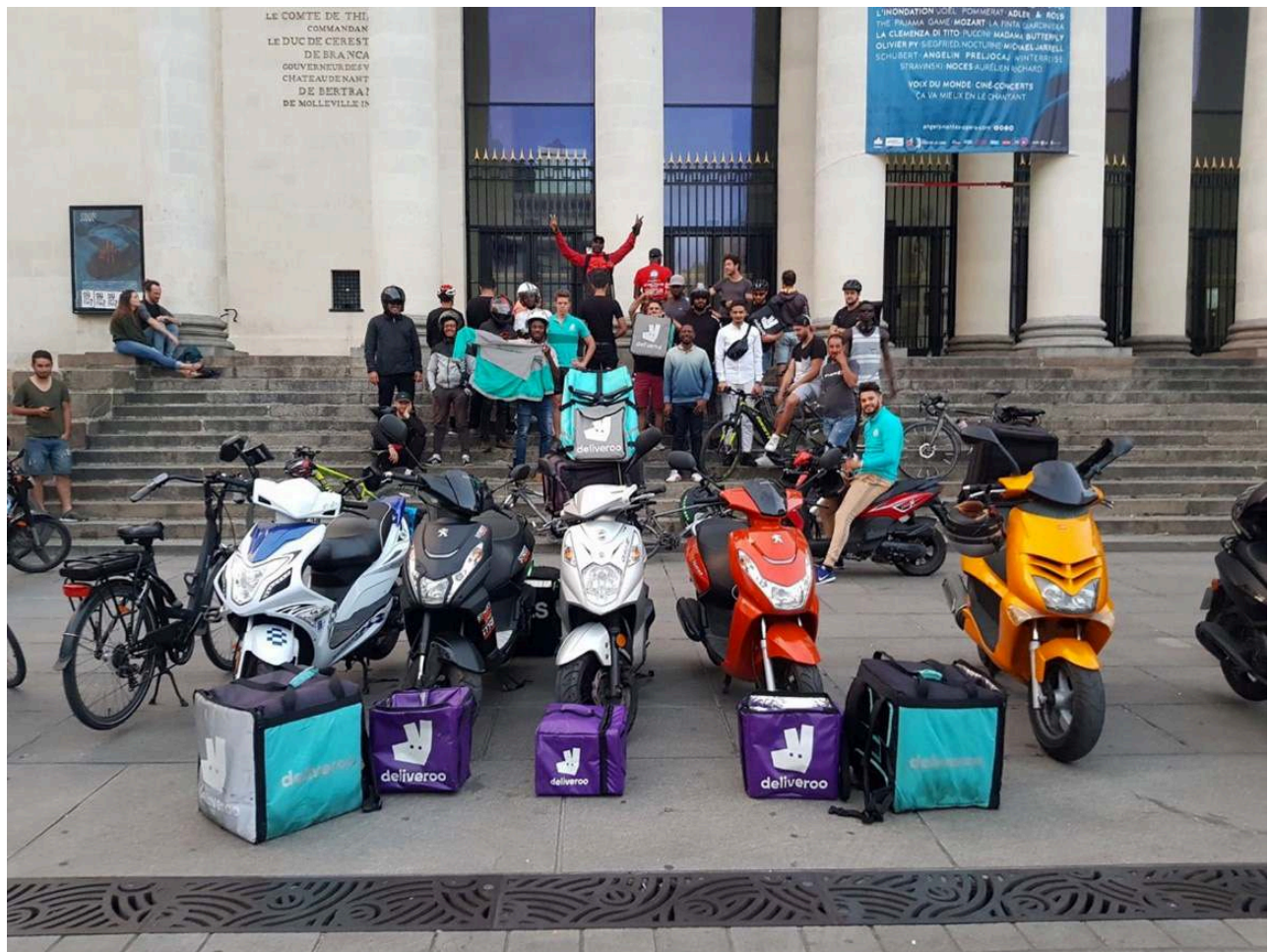

Crédit : Dorian Chupin

Source : Page Facebook du Syndicat des Coursiers Autonomes de Loire-Atlantique, Nantes, août 2019

Cette photographie de mobilisation permet au passage de souligner les différences entre coursiers, à la fois entre couleur de peau mais aussi en termes d'équipement. Quand certains exercent en scooter, d'autres choisissent le vélo; la taille de leurs sacs est également variable.

Photographie 7. Mobilisations de coursiers bordelais en août 2019.

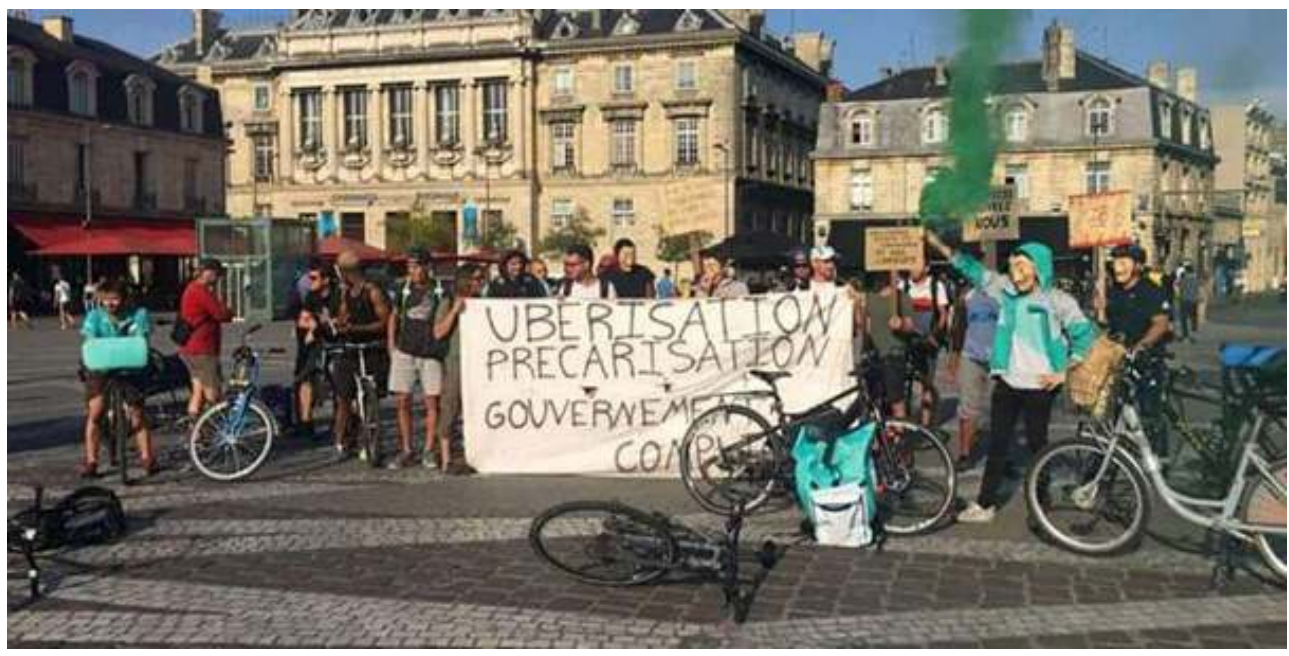

Source : site Facebook du syndicat CGT des coursiers à vélo de la Gironde, Bordeaux, août 2019. Crédit : libre de droits. 
Photographie 8. Mobilisations des travailleurs sans-papiers en juillet 2020.

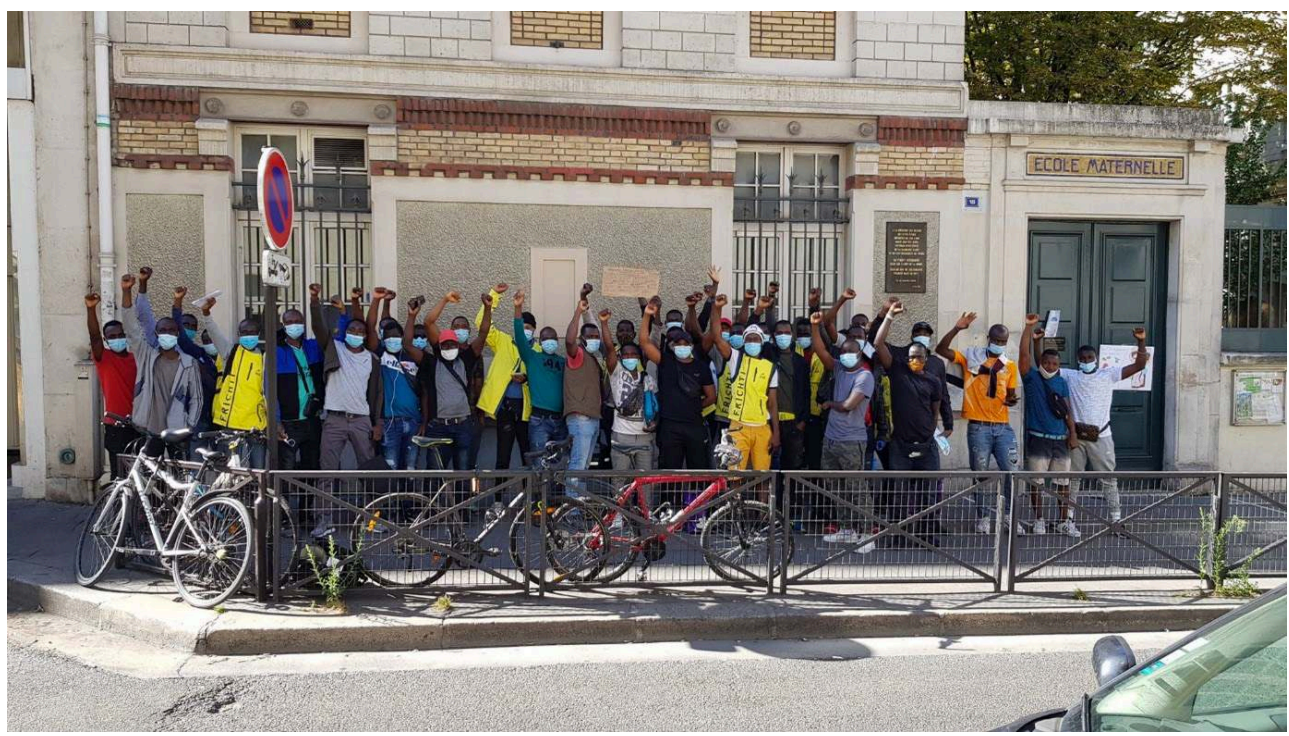

Source : page Facebook du CLAP, Collectif des Livreurs Autonomes de Paris, Paris, juillet 2020. Crédit : Jérôme Pimot

Ces mobilisations, qui ont fait l'objet d'un traitement médiatique important, ont déjoué toutes les logiques sociales qui conduisaient à maintenir ces travailleurs dans l'ombre et ont permis au contraire de les rendre ostensibles, alors même qu'un ensemble de caractéristiques rendait leur action collective peu probable (Collovald, Mathieu, 2009) : travailleurs indépendants micro-entrepreneurs, précarisés et mis en concurrence, hétérogénéité des usages de l'activité et des profils des coursiers, absence d'organisation collective, injonctions à la discrétion et même irrégularité de leur présence en France pour certains ${ }^{20}$. Ces travailleurs, se constituant progressivement en collectifs et par endroits soutenus syndicalement, se sont ainsi réapproprié leur espace de travail pour le transformer en espace de revendications et sortir des mécanismes d'invisibilisation. Usant de répertoires d'action (Tilly, 1986) relativement ordinaires tels que les rassemblements et l'arrêt de travail (ici le refus de commande), le temps de la mobilisation est néanmoins extraordinaire : leur visibilité n'est plus circonscrite à la rue mais « envahit » désormais de nouveaux espaces : politique, syndical et médiatique.

\section{Conclusion. Un confinement qui accentue la visibilité}

50 Parce qu'ils travaillent dans l'espace public, la visibilité des coursiers est, pour l'œil de l'observateur, patente lorsqu'ils attendent leur commande et plus furtive lorsqu'ils optimisent le temps de la course. Pourtant, elle est loin d'être une évidence tant elle est l'objet d'enjeux de reconnaissance sociale du labeur et de l'existence-même de ces «nouveaux valets» (Gorz, 1990). En ce temps de confinement décidé pour limiter la propagation de la pandémie de Covid-19 - durant lequel est écrit cet article - leur présence est pourtant flagrante: alors que les espaces publics sont désertés, les coursiers sont, avec les policiers et les éboueurs, les seuls travailleurs à y demeurer actifs. Le confinement rend d'autant plus manifestes les inégalités sociales qu'il les accroit : parmi les coursiers, ceux qui restent dans la rue sont ainsi les plus précaires, les plus dépendants économiquement à l'activité, et aussi les "moins blancs ». La photographie 9 permet de faire ce constat. Prise à Poitiers le 8 avril 2020 à $12 \mathrm{~h} 08$, à 
une heure où les coursiers sont habituellement particulièrement nombreux sur la place, elle témoigne de ce fait: les coursiers y sont nettement moins nombreux que d'ordinaire, ils sont tous noirs.

Photographie 9. Des coursiers attendent leur commande pendant le confinement.

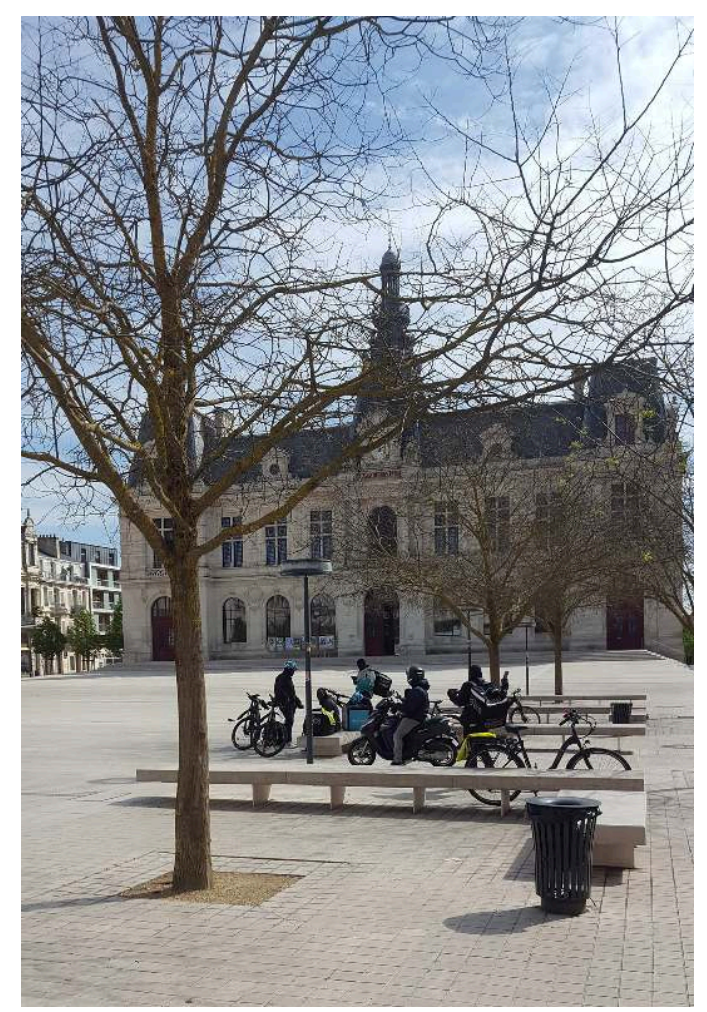

Crédit : Hélène Stevens, Poitiers, le 8 avril 2020 à 12 h 08, pendant le confinement.

51 Mais la tension entre visibilité et invisibilité n'a pas disparu pour autant. En effet, des consignes spécifiques ont été définies le 15 mars 2020 par le gouvernement et les plateformes, pour que « la livraison de repas à domicile reste autorisée pourvu qu'elle se fasse sans contact ${ }^{21}$. Ainsi, il est exigé des coursiers qu'au moment de la livraison au client, ils préviennent ce dernier, déposent le repas et repartent immédiatement sans avoir à croiser quiconque. Le "devoir d'invisibilité » (Leblanc, Cuvillier, 2020) est ici explicitement formulé. Dans les faits, les coursiers sont pris dans une contrainte paradoxale: ils doivent concilier cet impératif social et sanitaire et celui d'une satisfaction des clients qui parfois protestent lorsque la livraison se fait en bas de l'immeuble (Le Lay, Lemozy, 2020, 3).

Enfin, l'une des traductions exacerbées de ces inégalités sociales durant cette période de Covid-19 se donne à voir dans la campagne de remerciements adressés par les joueurs du PSG aux coursiers Deliveroo, entreprise sponsor du club parisien depuis septembre 2019. Une campagne vidéo montre ainsi les joueurs du PSG remerciant les livreurs pour leur travail pendant la période de confinement. 
Photographies 10 et 11 . Campagne de remerciements de Deliveroo en partenariat avec le PSG adressée aux livreurs pendant le confinement (avril 2020).
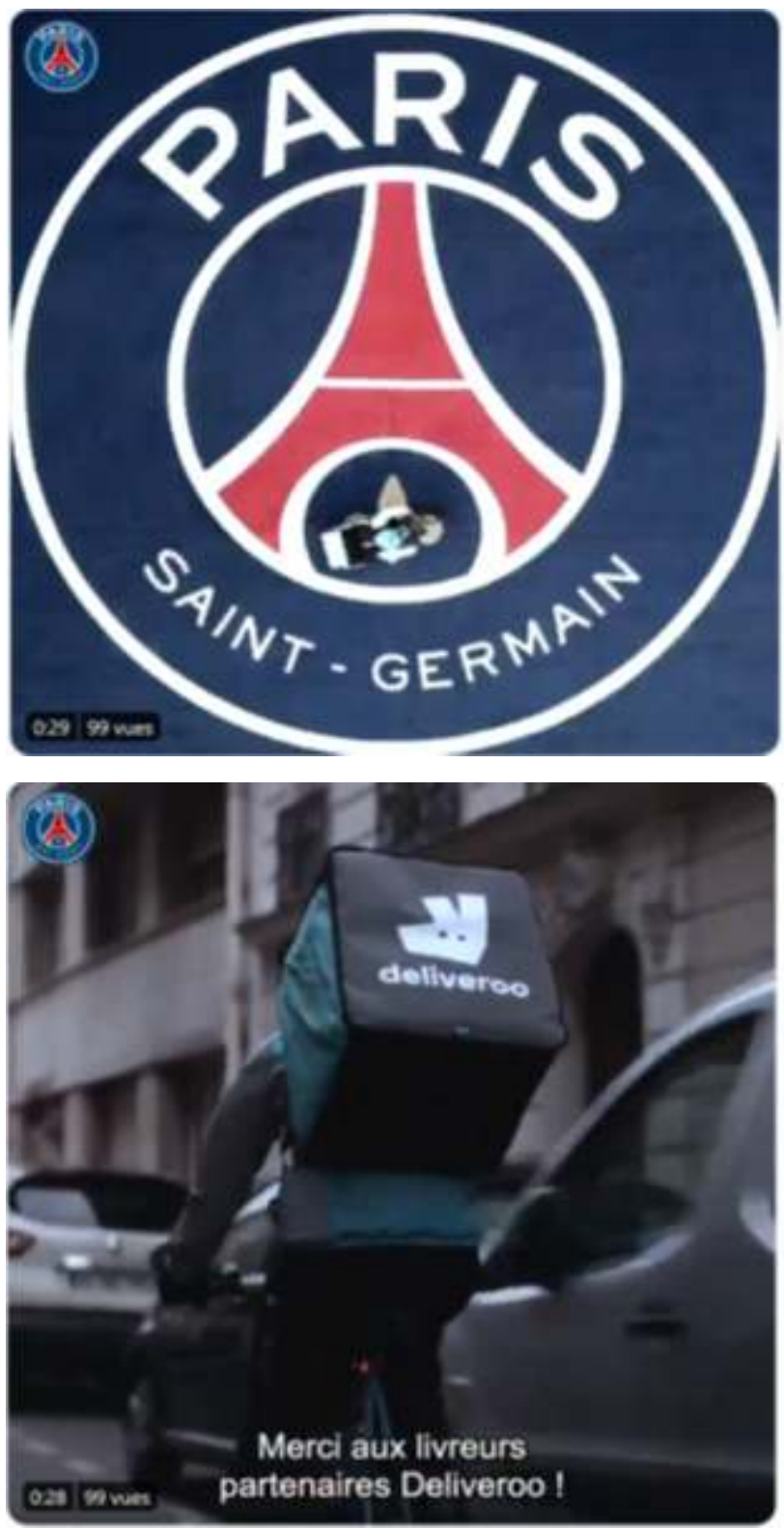

53 L'image 10 montre le drapeau du PSG sur lequel un coursier semble circuler. L'image 11 met en scène un coursier en train de livrer tandis que le symbole du PSG est apposé en haut de l'écran. La campagne publicitaire vise à féliciter les coursiers pour leur mobilisation en période de confinement, mais cette forme d'héroïsation soudaine et ponctuelle est une façade puisque les modifications organisationnelles opérées par les plateformes en cette période ${ }^{22}$ s'apparentent davantage à «un désaveu de reconnaissance » (Le Lay, Lemozy, 2020, 3).

Mais cette action n'est pas sans contradiction: les footballeurs, figures de réussite sociale et économique pour une partie des jeunes immigrés ou enfants d'immigrés, restent à l'abri pendant que leurs aficionados sont au travail dans la rue. La sécurité financière des premiers les préserve de l'exposition au virus, la dépendance économique des seconds les y confronte directement. 


\section{BIBLIOGRAPHIE}

Abdelnour S. et Méda D. (2019) Les nouveaux travailleurs des applis, Paris, PUF.

Aguilera A., Dablanc L. et Rallet A. (2018) «L'envers et l'endroit des plateformes de livraison instantanée. Enquête sur les livreurs micro-entrepreneurs à Paris ", Réseaux, vol. 212, n 6, p. 23-49.

Assegond C. (2012) «Les débuts de la photographie du travail usinier : production, représentations, usages (1870-1915) », dans Géhin J.-P., Stevens H., Images du travail, Travail des images, PUR, p. 87-100.

Aunis E. et Stevens H. (2020) « Usages sociaux de l'activité de coursiers de plateforme numérique ", Communication aux journées d'études européennes « La sécurité sociale des travailleurs des plateformes », 27 janvier, Université de Bordeaux.

Avril C., Cartier M. et Serre D. (2010) Enquêter sur le travail. Concepts, méthodes, récits, La Découverte.

Bernard S. (2013) «Travailler “à l'insu” des clients. Défaut de reconnaissance en caisses automatiques ", Travailler, $\mathrm{n}^{\circ}$ 29, p. 119-139.

Casilli A. (2019) En attendant les robots. Enquête sur le travail du clic, Editions du Seuil.

Chevalier L. (1958) Classes laborieuses et classes dangereuses à Paris pendant la première moitié du XIX ${ }^{e}$ siècle, Paris, Plon.

Corteel D. et Le Lay S. (2011) « Introduction. Travailler aux abords des déchets : un clair-obscur contemporain ", dans Corteel, Delphine, et Stéphane Le Lay, Les travailleurs des déchets, Toulouse, ERES, p. 15-32.

Collovald A. et Mathieu L. (2009) « Mobilisations improbables et apprentissage d'un répertoire syndical », Politix. Revue des Sciences Sociales du Politique, n 86, p. 119-143.

Gaborieau D. (2017) «Quand l'ouvrier devient robot. Représentations et pratiques ouvrières face aux stigmates de la déqualification ", L’Homme \& la Société, vol. 205, n 3, p. 245-268.

Goffman E. (1975) Stigmate : les usages sociaux des handicaps, Minuit, Paris.

Gorz A. (1990) « Pourquoi la société salariale a besoin de nouveaux valets », Le Monde Diplomatique, p. 22-23.

Harzfeld N. (2002) « La pause casse-croûte. Quand les chaînes s'arrêtent à Peugeot-Sochaux », Terrain, $n^{\circ} 39$, p. 33-48.

Jan A. (2018) «Livrer à vélo... en attendant mieux », La nouvelle revue du travail [En ligne], 13, mis en ligne le 29 octobre 2018, consulté le 04 août 2020. URL : http://journals.openedition.org/nrt/ 3803

Jounin N. (2004) «L'ethnicisation en chantiers. Reconstructions des statuts par l'ethnique en milieu de travail ", Revue européenne des migrations internationales, vol. 20 - n 3, p. 103-126.

Krinsky J. et Simonet M. (2012) « Déni de travail : l'invisibilisation du travail aujourd'hui », Sociétés contemporaines, $\mathrm{n}^{\circ} 87$.

Lebas C. (2019) « Carrière d'auto-entrepreneur et rapports (critiques) au travail : comment les coursiers à vélo font émerger des contestations ", La Revue de l'Ires, vol. 99, n 3, p. 37-61. 
Lejeune, G. (2019) « La dimension ethnique dans le travail des chauffeurs de taxi parisiens », Emulations, en ligne. Mise en ligne le 8 février 2019. DOI : 10.14428/emulations.varia.022

Lemozy F. (2019) « La tête dans le guidon », La nouvelle revue du travail [En ligne], 14|2019, mis en ligne le 07 mai 2019, URL : http://journals.openedition.org/nrt/4673.

Le Lay S., Lemozy F. (2020) «La banalisation du recours aux plateformes. Le cas des livreurs à domicile en période de pandémie », Contretemps, décembre ? https://www.contretemps.eu/ travail-plateformes-pandemie-covid-livreurs-deliveroo/.

Leblanc E., Cuvillier B. (2020) « Reconnus exposés. Le coursier à vélo et le choix cornélien », Anact, La revue des conditions de travail, $\mathrm{n}^{\circ} 10$.

Linhart D. (2015) La comédie humaine du travail. De la déshumanisation taylorienne à la surhumanisation managériale, Paris, Erès.

Memmi D. (2019) « Servir (chez) les autres. Pérennité et mutations de la domination rapprochée ", Actes de la recherche en sciences sociales, vol. 230, n 5, p. 108-119.

Molinier P. (2010) « Au-delà de la féminité et du maternel, le travail du care », Champ psychosomatique, 58, p. 161-174.

Nerrière X. (2016) «Conscience politique et conscience photographique », Images du travail Travail des images [En ligne], Images du travail, Travail des images, $\mathrm{n}^{\circ} 2$. Les ouvriers et la photographie : de 1945 à nos jours, Dossier, mis à jour le : 24/06/2016, URL : https://journals.openedition.org/itti/ 1151.

Paugam S. (2009) La disqualification sociale. Essai sur la nouvelle pauvreté. Presses Universitaires de France.

Raibaud Y. (2015) Une ville faite par et pour les hommes, Belin, coll. «Égale à égal ».

Roy D. (2006) Un sociologue à l'usine, Paris, La Découverte.

Savignac E. (2017) La gamification du travail. L'ordre du jeu, Londres, ISTE éditions, coll. «Innovation, entrepreneuriat et gestion ».

Sayagh D., (2017) «Construction sociospatiale de capabilités sexuées aux pratiques urbaines du vélo », Les Annales de la recherche urbaine, $\mathrm{n}^{\circ}$ 112, « Le genre urbain », p. 126-137.

Soares A. (2011) « L'élégance des éboueurs », dans D. Corteel (éd.), Les travailleurs des déchets, Toulouse, ERES, p. 213-234.

Tilly C. (1986) La France conteste, de 1600 à nos jours, Fayard.

Tissot S. (2012), « Les centres-villes : modèles, luttes et pratiques », Actes de la recherche en sciences sociales, $\mathrm{n}^{\circ} 195$, p. 4-11.

Tourneur L. (2019) « Un travail à ciel ouvert » Ethnographie de coursiers de plateformes numériques, mémoire de master 1 , sous la direction d'H. Stevens, Université de Poitiers.

\section{NOTES}

1. Cette contribution s'appuie sur les résultats du programme de recherche financé par la DreesMire, «Formes de mobilisation collectives et économie des plateformes» coordonné par I. Daugareilh, convention de recherche $\mathrm{N}^{\circ} 2102608269$ - janvier 2019-octobre 2021. Les observations de même que les entretiens (exceptés ceux avec les travailleurs sans-papiers mobilisés à Paris) ont été réalisés à Poitiers et à Bordeaux. L'étendue, la topographie et la densité 
démographique de la ville peuvent agir sur certaines modalités de l'activité et leur gestion par les coursiers. Par exemple, les dénivelés importants à Poitiers imposent aux coursiers de trouver des combines pour les éviter au maximum. Pour autant, un ensemble de phénomènes ici décrits est repérable dans les deux villes.

2. Nous remercions Henri Eckert et Benjamin Néa de nous avoir confié leurs prises de vue de coursiers. Merci également à Lisa Tourneur pour avoir partagé avec nous de riches moments d'échanges sociologiques ainsi que ses matériaux d'enquête dans le cadre de son mémoire de master (Tourneur, 2019).

3. Pour respecter l'anonymat des enquêtés, l'ensemble des prénoms a été modifié.

4. Les contrôles par Uber Eats se sont toutefois progressivement renforcés, puisque la plateforme a d'une part refusé les étudiants étrangers à partir de juillet 2018 et d'autre part mis en en place en 2020 un système de reconnaissance faciale qui impose aux coursiers de se prendre en photo avant de se connecter. Ce contrôle s'exerce de manière aléatoire et incite certains livreurs sans papiers à travailler plutôt avec la plateforme Deliveroo.

5. Sur ce sujet, nous pouvons mentionner le travail d'enquête réalisé par un journaliste de Libération (et publié dans trois articles parus les 3, 4 et 5 novembre 2020 : " "Loue un compte Uber Eats, 100 euros par semaine" : enquête sur la livraison en sous-location »; "Avant j'étais vendeur de tours Eiffel: enquête sur la livraison en sous-location" et "Livreurs sans papiers, sous locations: "C'est un sujet qui dépasse Stuart" ", de Gurvan Kristanadjaja), ainsi que celui de journalistes de Ouest-France ( $«$ Location de compte, repas, appart... Comment les réseaux exploitent les livreurs sans-papiers » et «Pourquoi y a-t-il tant de jeunes Guinéens parmi les livreurs de repas à domicile? publiés le 27/01/2021).

6. En France, une décision du 13 décembre 2017 de la cour d'appel de Paris a requalifié en salarié un chauffeur VTC indépendant travaillant pour la plateforme Le-Cab. Par la suite, l'arrêt de la Cour de cassation du 28 novembre 2018 a reconnu un travailleur de Take Eat Easy comme salarié. Cet arrêt a été suivi du jugement de la cour d'appel de Paris le 10 janvier 2019, puis de l'arrêt du 4 mars 2020 de la Cour de cassation qui ont reconnu comme salariés des chauffeurs VTC Uber.

7. Les coursiers voient sur la carte les différents restaurants où il peut y avoir des courses. Ici Julien a tenté une première fois de se rendre à ce restaurant peu connu et a fait le constat qu'il recevait ainsi beaucoup de courses, souvent courtes, lui permettant de nombreux allers-retours.

8. Cette figure de coursier est d'ailleurs celle qui est mobilisée dans les campagnes publicitaires et de recrutement des plateformes.

9. Ces «bikers» peuvent vivre les temps d'attente avec moins de tension, comme Célestin (24 ans, étudiant en $3^{\mathrm{e}}$ cycle de conservatoire, Deliveroo, $\sim 4 \mathrm{~h} /$ semaine) qui en profite pour lire ou appeler des proches et qui dit "J'prends le temps pour moi en fait. »

10. En effet, sur les $38 \%$ de jeunes âgés entre 12 et 17 ans qui déclarent faire du vélo, $61 \%$ sont des garçons et $39 \%$ des filles (ministère des Sports [2002], Sports STAT-Info, Bulletin de statistiques et d'études, $\mathrm{n}^{\circ}$ 02-04), et à l'âge adulte, parmi les personnes ayant pratiqué du vélo au cours des quatre dernières semaines, 58 \% sont des hommes et $42 \%$ des femmes («Pratiques physiques ou sportives des femmes et des hommes: des rapprochements mais aussi des différences qui persistent », Insee Première, $\mathrm{n}^{\circ} 1675$, 2017)

11. Le BMX, «bicycle moto cross », désigne soit une course de compétition sportive de « supercross » sur des pistes parsemées de bosses, soit des pratiques sportives urbaines consistant à effectuer des figures techniques et spectaculaires (le "freestyle »); dans les deux cas, le vélo utilisé est petit, léger, maniable, avec peu d'équipement. Le fixie quant à lui renvoie à un vélo sans frein et dont le pignon de la roue arrière est fixe, i.e., sans roue libre et à une seule vitesse, ce qui permet de maximiser le rendement du coup de pédale. Initialement utilisé par les coureurs cyclistes sur piste et, bien qu'il soit formellement interdit par le Code de la route, il est aujourd'hui apprécié des adeptes de vélo urbain, inspirés des pratiques des «bikes-messengers ", coursiers des grandes villes étatsuniennes des années 1990. 
12. Sayagh D., (2017), «Construction sociospatiale de capabilités sexuées aux pratiques urbaines du vélo », Les Annales de la recherche urbaine, n 112, Le genre urbain. p. 126-137.

13. On y retrouve alors cet aspect de « la double vérité du travail » qu'analysait Pierre Bourdieu : «Les travailleurs peuvent concourir à leur propre exploitation, par l'effort même qu'ils font pour s'approprier leur travail et qui les attache à lui par l'intermédiaire des libertés, souvent infimes et presque toujours "fonctionnelles", qui leur sont laissées. » in Pierre Bourdieu, «La double vérité du travail », dans Méditations pascaliennes, Paris, Seuil, 1997, p. 241-242.

14. Ou alors de protester (cf. partie 3), d'arrêter l'activité de coursier ou encore de la pratiquer en dehors des plateformes.

15. Le taux d'acceptation/refus de commande des livreurs est un indicateur explicite d'évaluation de leur activité dans l'application de la plateforme et donne lieu à des interprétations variées sur les possibilités de sanctions par les plateformes s'il n'est pas optimal. Dans l'incertitude sur son rôle effectif, la plupart des coursiers cherchent à le maintenir au plus haut.

16. Le niveau du SMIC est très souvent mentionné comme référence, avec l'enjeu de le dépasser, même si les calculs omettent souvent d'opérer la distinction entre chiffres d'affaires et revenus.

17. Le développement des plateformes est d'ailleurs régulièrement traité par les médias sur le registre de l'envahissement (ex. «Depuis plusieurs mois, les livreurs à vélo de repas envahissent les grandes villes, comme les VTC avant eux », Damien Licata Caruzo, "Deliveroo va proposer des assurances à ses livreurs à vélos », Le Parisien, 20 mars 2017 ; Delphine Girard, « La livraison à vélo de repas à domicile envahit Toulouse", France 3 Occitanie, 20 octobre 2017). Ce terme d'envahissement se définit comme le fait d'«occuper (un territoire) brusquement et par la force» (https://dictionnaire.lerobert.com), et contient deux caractéristiques: la célérité du phénomène et la violence. Dans le cas des coursiers, leur nombre a effectivement crû de manière importante et rapide, et l'origine sociale populaire et africaine d'une fraction d'entre eux a perturbé les hiérarchies socio-spatiales entre centre-ville et périphéries, rendant visible «le monde honni des « banlieues », « jeunes » et « immigrés » (Tissot, 2012, 11).

18. Cette consultation a été menée par la plateforme Uber en mars 2019 par la passation de 14000 questionnaires ( 5400 répondants) et d'une quarantaine de tables rondes organisées dans plusieurs villes de France. Elle a fait l'objet d'un rapport rendu public qui détermine sept axes que la société Uber souhaite développer: https://www.uber.com/fr/blog/actions-postconsultation-coursiers/.

19. Les transformations tarifaires imposées le sont toujours au cours de la période estivale, pendant laquelle l'activité se tarit et le nombre de coursiers en activité se raréfie.

20. La dernière mobilisation en date est à ce titre exemplaire puisqu'elle émane de travailleurs sans papiers se mobilisant pour leur réintégration à la plateforme Frichti. Elle fait suite à la parution d'un article de presse dans Libération le $1^{\mathrm{er}}$ juin 2020 "Sans papiers, sans contrat... bienvenue chez Frichti» relatant la mobilisation par la plateforme de travailleurs microentrepreneurs sans papiers. À la suite de cette parution, les contrôles ont été renforcés et les coursiers ont été tenus de présenter un titre de séjour autorisant l'exercice d'une activité indépendante. Dès lors, une grande partie des coursiers n'était plus en mesure de travailler pour cette plateforme. Plusieurs mobilisations ont eu lieu, sur la base de deux revendications principales: la réintégration à la plateforme et la régularisation sur le sol français. Les négociations entre la CGT, les leaders du mouvement et l'Etat ont donné lieu à un accord le 22 juillet 2020 permettant à la moitié des travailleurs d'accéder aux procédures de régularisation. Quatre mobilisations ont eu lieu depuis, menées par ceux qui n'ont pu y accéder.

21. Guide des précautions sanitaires à respecter dans le cadre de la livraison de repas à domicile ", 15 mars 2020. https://www.economie.gouv.fr/coronavirus-guide-des-precautionssanitaires-livraison-repas.

Images du travail, travail des images, 11 | 2021 
22. La plateforme Deliveroo est passée de créneaux à sélectionner à des «free shifts » en période de confinement, entraînant une concurrence accrue des coursiers et la baisse de leurs rémunérations.

\section{RÉSUMÉS}

À partir d'une enquête fondée sur des observations et entretiens, cet article étudie les pratiques de travail des coursiers dans l'espace public, polarisées entre un temps improductif d'attente non dénué de formes de rationalisation de l'activité et durant lequel s'engagent des sociabilités entre pairs - et un temps rémunérateur de livraison - qui donne lieu à des stratégies d'optimisation impliquant résistance physique, prises de risques et logiques de ménagement. Entre patienter sur les places et foncer dans les rues, ces travailleurs sont pris dans diverses tensions, dont une, centrale, est celle qui se joue entre visibilité de leur présence dans l'espace public et invisibilisation de leur travail.

Based on a survey mixing observations and interviews, this article studies the working practices of couriers in the public space. These practices, polarized between an unproductive time of waiting-not devoid of forms of rationalization of the activity and during which sociabilities between peers are engaged-and a remunerative delivery time-which gives rise to optimization strategies involving physical resistance, risk-taking and logics of moderation. Between waiting in the squares and rushing through the streets, these workers are caught up in various tensions, one of which is the central one between the visibility of their presence in the public space and the invisibility of their work.

\section{INDEX}

Mots-clés : coursiers, travailleurs des plateformes numériques, invisibilisation, accélération, rapports sociaux

Keywords : couriers, digital platform workers, invisibility, acceleration, social relations

\section{AUTEURS}

\section{ÉMILIE AUNIS}

Émilie Aunis est postdoctorante à l'Université de Bordeaux (Comptrasec) au sein du programme de recherche NUTRA l'« Economie des plateformes et les mutations du travail et des organisations ». Ses travaux portent sur les transformations des formes d'emploi et des conditions de travail. Sa thèse soutenue en 2014 qui s'intitule D'improbables ouvriers? Les ouvriers d'industrie poitevins, entre dissolution et recomposition des collectifs analysait les transformations du travail ouvrier, et l'altération des collectifs ouvriers tout en interrogeant les ressorts de l'action collective ouvrière rendue à bien des égards improbables. Elle a ensuite travaillé comme chercheure postdoctorale à l'université Laval à Québec pendant deux ans dans le cadre d'une recherche collective sur Les nouvelles configurations de la relation d'emploi et leurs impacts sur le 
travail, l'emploi et l'action collective sur deux secteurs d'activité (les chauffeurs Uber, l'industrie du matériel de transport).

\section{HÉLĖNE STEVENS}

Hélène Stevens est maîtresse de conférences à l'Université de Poitiers (GRESCO). Ses travaux analysent la figure contemporaine de l'individu comme « entrepreneur de soi » en enquêtant de manière conjointe sur les formes de diffusion, réception et usage d'une culture de développement personnel et sur le regain et les transformations du travail indépendant (description du champ de l'encouragement à la création d'entreprise, suivi de microentrepreneurs, recherche pluridisciplinaire sur les travailleurs de plateformes numériques). Elle est co-autrice de Les sciences sociales et Bourdieu avec Wenceslas Lizé et Catherine Leclerc, paru à La Dispute (2015), de « Les cadres pris dans la gestion », Savoir/Agir, avec Laurent Willemez (vol. $40, n^{\circ} .2,2017$ ) et de «Les passeurs de l'entrepreneuriat à l'université. Incertitudes et tensions autour d'un espace professionnel émergent ", Actes de la Recherche en Sciences sociales, avec Olivia Chambard (n²39, 2021). 